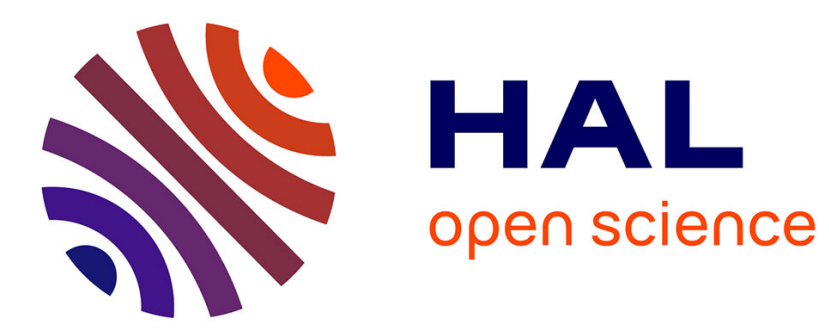

\title{
Discharge of a silo through a lateral orifice: Role of the bottom inclination versus friction
}

Z Zou, Pierre Ruyer, P.-y Lagrée, Pascale Aussillous

\section{To cite this version:}

Z Zou, Pierre Ruyer, P.-y Lagrée, Pascale Aussillous. Discharge of a silo through a lateral orifice: Role of the bottom inclination versus friction. Physical Review E , 2020, 102, pp.052902. 10.1103/PhysRevE.102.052902 . hal-03023960

\section{HAL Id: hal-03023960 https://hal.science/hal-03023960}

Submitted on 25 Nov 2020

HAL is a multi-disciplinary open access archive for the deposit and dissemination of scientific research documents, whether they are published or not. The documents may come from teaching and research institutions in France or abroad, or from public or private research centers.
L'archive ouverte pluridisciplinaire HAL, est destinée au dépôt et à la diffusion de documents scientifiques de niveau recherche, publiés ou non, émanant des établissements d'enseignement et de recherche français ou étrangers, des laboratoires publics ou privés.

$$
\text { Copyright }
$$




\title{
Discharge of a silo through a lateral orifice: Role of the bottom inclination versus friction
}

\author{
Z. Zou, ${ }^{1,2}$ P. Ruyer $\odot,{ }^{1}$ P.-Y. Lagrée, ${ }^{3}$ and P. Aussillous $\odot^{2, *}$ \\ ${ }^{1}$ Institut de Radioprotection et de Sûreté Nucléaire (IRSN), PSN-RES, SEMIA, LSMA, Cadarache, St. Paul-Lez-Durance 13115, France \\ ${ }^{2}$ Aix-Marseille Université, CNRS, IUSTI, Marseille, France \\ ${ }^{3}$ Sorbonne Université, CNRS UMR7190, Institut Jean le Rond $\partial^{\prime}$ Alembert, F-75005 Paris, France
}

(Received 10 March 2020; accepted 2 October 2020; published 9 November 2020)

\begin{abstract}
In this work we propose to identify the relative role of the inclination of gravitational acceleration and friction on the discharge flow rate of a granular media from a rectangular silo by varying the silo geometry thanks to an inclined bottom which ends up at a lateral outlet. The study is motivated by a nuclear safety problem: a fuel rod (modeled by an elongated silo) accidentally releases fuel fragments (modeled by grains). We performed experiments where we independently measured the mass flow rate and the velocity profiles, together with discrete particle simulations and continuum simulations with a frictional rheology described by a $\mu(I)$ constitutive law and taking into account the wall friction. We study monolayer flows and three-dimensional flows, and we propose an analytical model that predicts the discharge flow rate of particles from a rectangular silo with an inclined bottom according to its outlet aspect ratio and the bottom inclination angle.
\end{abstract}

DOI: 10.1103/PhysRevE.102.052902

\section{INTRODUCTION}

Silos are widely used in industry, and empirical predictions of flow rate, based on scaling law, have existed for more than a century [1-3]. Historically, the Hagen-Beverloo relation was explained with the concept of a "free-fall arch" located at the outlet of diameter $D$ from which the particles fall freely, leading to a velocity $v_{0} \sim \sqrt{g D}$ at the outlet and a mass flow rate

$$
Q \sim \phi_{b} \rho_{p} \sqrt{g D^{5}}
$$

(with $\phi_{b}$ the bulk volume fraction and $\rho_{p}$ the particle density). This relation does not depend on the exact cross-section shape of the silo, either a circle of radius $L$ or a square of length $L$. In the case of a rectangular silo of width $L$ and of thickness $W$ [4,5], with an orifice of size $D$ spanning with the thickness (see Fig. 1), it leads to a mass flow rate (consistent with dimensional analysis [6])

$$
Q=c_{D} \phi_{b} \rho_{p} W \sqrt{g D^{3}},
$$

where $c_{D}$ is a coefficient without dimension. Thanks to the development of new experimental techniques and numerical simulations, this "free-fall arch" concept has been recently questioned. Experimentally and with discrete simulations, it has been shown $[4,7]$ that the granular medium remains dense and that the particles do not exactly undergo a free fall but accelerate on a zone located at the outlet with a small dilation. Moreover, continuum modeling with the so-called $\mu(I)$ viscoplastic frictional rheology, based on a local constitutive law between the local shear rate and the stress tensor [8], have been successfully used to recover the Hagen-Beverloo scaling as in Refs. [9-11], with very different numerical methods.

\footnotetext{
*pascale.aussillous@univ-amu.fr
}

Another way to test this concept is to consider nonconventional geometry, for example, tilted silos [12] or silos with a lateral orifice [6]. This last case has practical interest in the context of safety studies of nuclear power plants. Considering a hypothetical accident scenario, namely, a beyond design basis control rod ejection, it would lead to the failure of the cladding of some fuel rods and the ejection of fuel fragments. The flow of fuel fragments determines the intensity of some of the consequences of such failure: ejection of hot fuel fragments within the reactor coolant flow leads to a violent thermal interaction whose intensity scales with this flow rate. The fuel fragments can be idealized as a granular media and the fuel rod as an elongated silo. Due to the accident, the lateral opening of the rod has a shape which is not a circle of size $D$ but which is idealized by a rectangular shape. This simplified analogy has motivated this study. Interestingly, in these geometries the Hagen-Beverloo scaling is seen to remain valid, except for narrow rectangular silos $(W \ll D)$, where a new regime of flow appears [6]. In this paper it has been shown that these two regimes of discharge can be simulated thanks to a continuum modeling for the granular media with the frictional rheology $\mu(I)$ in two-dimensional (2D) computations where the wall friction force (along the front and rear walls of the silo) is accounted for from a Hele-Shaw point of view. In those simulations it was observed that $U_{0}$, the norm of the velocity on the central streamline, does not depend on the lateral friction:

$$
U_{0}=c_{E} \sqrt{g D}
$$

(where $c_{E}$ is a coefficient of order one), which suggests that the potential energy is transferred to kinetic energy in the outlet area, whatever the outlet position. Then it has been observed that the wall friction term mainly influences the orientation of the internal flow of the granular media. For lateral aperture, the granular flow, which is accelerated by the vertical gravity, has to rotate toward the aperture. The wall 


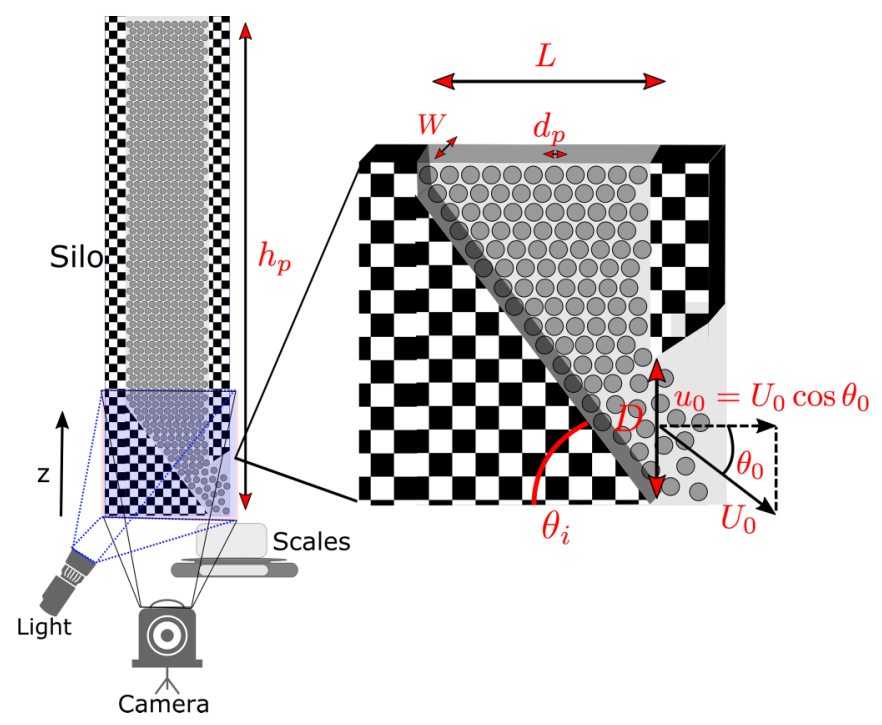

FIG. 1. Sketch of the experimental setup with a zoom on the aperture. Main discussed parameters in red: $d_{p}$ grain diameter, $W$ thickness of silo, $L$ width of silo, $h_{p}$ height of grains, $U_{0}$ velocity on the central streamline, $\theta_{0}$ inclination of the central streamline at the orifice of size $D$, and $\theta_{i}$ angle of inclination of the bottom wall. Gravity is aligned with but opposite to the $z$ axis.

friction term was shown to tend to align the flow toward the direction of gravity, therefore reducing the horizontal component of the flow velocity, normal to the aperture. Hence a reduction of mass flow rate was observed as well as a new regime controlled by the thickness of silo $W$. From the two limiting cases $W \gg D$ and $W \ll D$, Zhou et al. [6] proposed an empirical model to predict the mass flow rate as a function of the aperture aspect ratio,

$$
\frac{Q}{\rho_{p} \phi_{b} \sqrt{g W^{5}}}=c_{D} \frac{(D / W)^{3 / 2}}{\sqrt{1+\gamma D / W}},
$$

where $c_{D}$ is a numerical constant that can take into account the particle diameter, which seems to play a role only in the volume fraction around the aperture. This relation gives back Eq. (2) for $W \gg D$. Then, from this, the angle of inclination $\theta_{0}$ (see Fig. 1) of the central streamline at the orifice relative to the horizontal is estimated as

$$
\cos \theta_{0}=\frac{c_{\theta_{0}}}{\sqrt{1+\gamma D / W}},
$$

where $c_{\theta_{0}}$ and $\gamma$ are numerical constants.

The aim of this study is to validate and generalize these observations to better understand the physical processes leading to the Hagen-Beverloo scaling. Thus we propose to identify the relative role of the inclination of gravitational acceleration and the friction (internal or due to the wall) on the discharge flow rate of a granular media from a rectangular silo by varying the silo geometry thanks to an inclined bottom which ends up at a lateral orifice. The methods are presented in Sec. II. We performed experiments where we independently measure the mass flow rate and the velocity profiles. We also performed 2D discrete particle simulations and continuum simulations with a frictional rheology described by a $\mu(I)$ constitutive law and taking into account the wall friction with a Hele-Shaw point of
TABLE I. Geometrical parameters for the experimental performed runs where $D$ is the outlet size, $\theta_{i}$ the angle of inclination of the bottom wall, and $d_{p}$ the grain diameter. These parameters are sketched in Fig. 1.

\begin{tabular}{lccc}
\hline \hline$D(\mathrm{~mm})$ & $W(\mathrm{~mm})$ & $\theta_{i}(\mathrm{deg})$ & $d_{p}(\mu \mathrm{m})$ \\
\hline $10 ; 20 ; 30 ;$ & $5 ; 10$ & $0 ; 20 ; 40 ;$ & Glass: $190 ; 375 ; 538 ;$ \\
$40 ; 50$ & & $60 ; 70 ; 80$ & $762 ; 1129 ; 1347$ \\
& & Ceramic: 4170 \\
\hline \hline
\end{tabular}

view. In Sec. III we study the monolayer flow and compare the experimental results with the $2 \mathrm{D}$ discrete simulations. Thanks to the experimental observations of the flow properties at the outlet, we propose a simple analytical model to predict the discharge flow. Then we focus on the three-dimensional (3D) flow in Sec. IV, where we compare the experimental results with the continuum simulations and we deduce an analytical model that predicts the discharge flow rate of particles from a rectangular silo with an inclined bottom according to its outlet aspect ratio.

\section{METHODS}

\section{A. Experiments}

As shown in Fig. 1, the experimental setup consists of a rectangular silo (of height $H=1 \mathrm{~m}$, width $L=10 \mathrm{~cm}$, and thickness $W$ which can be varied) made of two thin vertical glass plates separated on the sides by Plexiglas plates. The bottom of the silo is inclined thanks to a triangular Plexiglas plate with an inclined angle $\theta_{i}$ to the horizontal, which can be varied from $0 \mathrm{deg}$ (corresponding to a horizontal bottom) to $80 \mathrm{deg}$. This inclined bottom plate ends up at the lateral orifice, and its vertical position delimits the orifice size $D$. The granular material consists of smooth spherical glass beads (density $\rho_{p}=2500 \mathrm{~kg} \mathrm{~m}^{-3}$ ) with different diameters $d_{p}$ from 190 to $1347 \mu \mathrm{m}$ (with a dispersion of $\pm 10 \%$ ). The whole set of geometrical data is given in Table I. We also use spherical ceramic beads (density $\rho_{p}=6000 \mathrm{~kg} \mathrm{~m}^{-3}$ ) with a diameter $d_{p}=4170 \mu \mathrm{m}$ in the thinner silo to study the flow of a monolayer case.

Once the silo is filled with a mass $m_{p}$ of particles, the column height $h_{p}$ is measured, giving the initial bulk particle volume fraction $\phi_{b}=2 m_{p} /\left[W \rho_{p} L\left(2 h_{p}-L \tan \theta_{i}\right)\right]$. Then the outlet is quickly opened manually and the grains falling out of the silo are collected in a vessel whose temporal evolution of the mass is recorded using an electronic scale (Mettler Toledo 6002S) with a precision of $0.1 \mathrm{~g}$ at $20 \mathrm{~Hz}$. Each experiment is repeated twice to validate the reproducibility of the process. The instantaneous mass flow rate is obtained by measuring the local slope of the mass versus time during $1 \mathrm{~s}$. We observe a steady-state regime of discharge for all the configurations explored, and the steady flow rate $Q$ is obtained by time averaging the instantaneous flow rate during this stationary state with the dispersion evaluated by one standard deviation.

The motion of particles at the front plate is recorded using a high-speed camera (Photron FASTCAM APX RS with a SIGMA zoom, 24-70 $\mathrm{mm}$ f2.8) with a spatial resolution of 
$1024 \times 1024$ at a rate of 250 frames per second. The test section is located at the bottom of the silo and is $15 \mathrm{~cm}$ wide to permit both good visualization of the flow around the outlet and good resolution of the particle imaging (see Figs. 4 and 12). It is illuminated by a lamp which has a continuous blue bright field (M470L2-C5 blue collimated LED). Particle velocities are measured by particle image velocimetry (PIV) using the MATLAB software DPIVSOFT [13]. In practice, this involves using a square interrogation region having a size $S_{i r}=32$ pixels, with 60 boxes in both the horizontal and vertical direction. The local particle displacement at each node for each pair of consecutive images is measured using a direct cross correlation. The velocities are built up by taking the average of nine pairs of consecutive images which are picked up every $0.04 \mathrm{~s}$ during the steady-state period of discharge. The error is given by one standard error. The spatial resolution of the measurement (given by the height of the interrogation region) is $\approx 5 \mathrm{~mm}$, while the velocity resolution (given by the choice of the image pairs) is $\approx 0.004 \mathrm{~m} / \mathrm{s}$. Due to spatial resolution, we are not able to obtain the velocity fields for the small particles $\left(d_{p}<500 \mu \mathrm{m}\right)$ and for the small outlet size $(D \cos \theta<5 \mathrm{~mm})$. From the velocity fields, we compute the streamlines for velocities of magnitude higher than $0.01 \mathrm{~m} / \mathrm{s}$ (see Figs. 4 and 12). With each experiment being done twice, we verify the repeatability of the method and remove data when the difference between the measured velocities at the center of the outlet for the same condition is more than double whereas the flow rate is the same. We also removed data for which the standard deviation of the velocity at the center of the outlet is higher than the magnitude of this velocity. For clarity, in most of the graphs only some error bars are shown.

In the monolayer case we also compute the particle volume fraction. Using a threshold value, the images are made binary. Then an image representing the particle volume fraction is obtained by averaging 100 consecutive images corresponding to $0.4 \mathrm{~s}$ during the stationary discharge period, as can be seen in Fig. 6. We scale the pixel value in this image by using the bulk volume fraction $\phi_{b}$ evaluated by averaging the image on a zone far from the outlet and initially measured independently using the column height. Finally, we average the value on the same boxes than for the PIV processing.

\section{B. Discrete simulations for 2D flows}

We simulated an infinite thickness configuration using the contact dynamics method implemented in the LMGC90 software [14]. With these simulations we aim to investigate a true 2D flow, without the effect of the back and front sidewalls. As shown in Fig. 2(a), the two-dimensional silo consists of a rectangular silo of width $L=3 D$ bounded by two vertical walls and an inclined bottom with a variable angle, $\theta_{i} \in[0 ; 20 ; 40 ; 60 ; 70]$ deg, which ends up at the right wall delimiting the lateral orifice of size $D$ which can be varied $(D \in[40 ; 45 ; 50 ; 55 ; 60] \mathrm{mm})$. The silo is initially filled using a random deposition with a height $h_{p}=18 D$ of spherical particles of mean size $d_{p}=4.2 \mathrm{~mm} \pm 10 \%$ (density $\rho_{p s}=100 \mathrm{~kg} \mathrm{~m}^{-3}$ ). We considered that the particles are perfectly rigid and inelastic, and their contact dissipation is modeled in terms of a friction coefficient that we set to
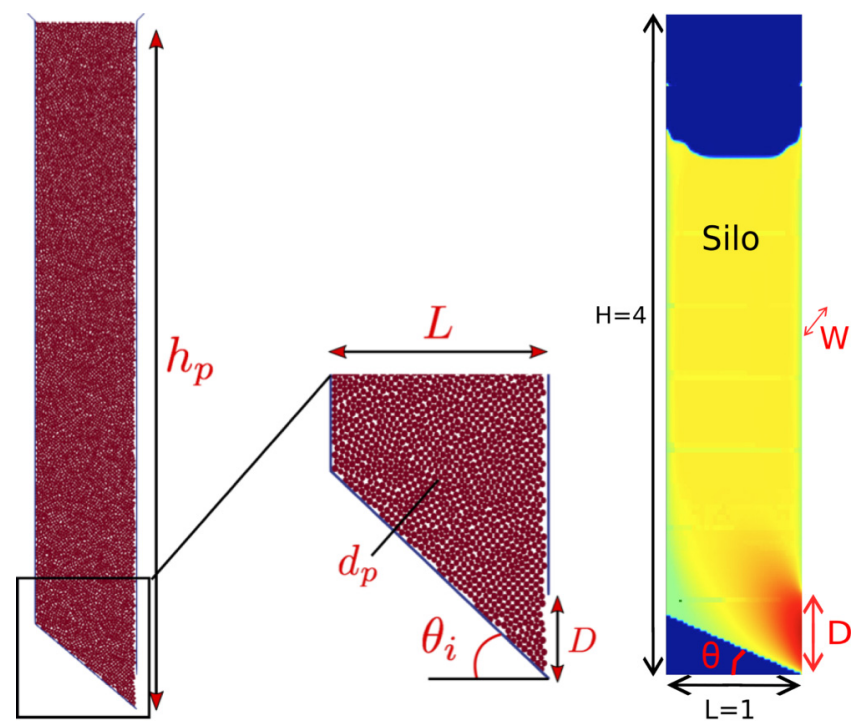

(a)

(b)

FIG. 2. Simulated silos with bottom inclination and lateral orifice: (a) 2D discrete simulations and (b) averaged Hele-Shaw continuum simulations.

$\mu_{p}=0.4$ between the particles and to $\mu_{w}=0.5$ between the particles and the walls [15].

For comparison with the laboratory experiments, the data are analyzed similarly to a monolayer flow in a silo of thickness $W=d_{p}$. After the preparation phase the initial bulk particle volume fraction was obtained by considering the surface average $\sigma$ of the particle indicator function (whose value is 1 on the particles and 0 otherwise) over the central zone of the silo with $\phi=2 \sigma / 3$. Then the simulations are run with a time step of $\delta t=5 \times 10^{-4} \mathrm{~s}$ for a number of time steps $N_{t}=8000$. The computational domain is periodic in the vertical direction to keep constant the number of particles (see [16] for more details). A snapshot of all particle positions and velocities was recorded every 20 time steps. From these snapshots the instantaneous flow rate was obtained by measuring the surface of particles $S_{p}$ leaving the silo during $\Delta t=0.2 \mathrm{~s}$ and calibrating with the experimental particle density: $Q_{i}=\rho_{p} / \rho_{p s}\left(\sum_{\Delta t} 2 S_{p} d_{p}\right) /(3 \Delta t)$. Similarly to the experiments, we observed a steady-state regime of discharge and we measure the mean flow rate $Q$ on this period.

To obtain the velocity and the volume fraction fields at the outlet, the outlet area of dimension $D \times d_{\max }$, where $d_{\max }$ is the maximum diameter of particles, is divided in several smaller rectangular boxes of length $a=0.2 d_{\max }$. Following the ergodicity theory, the velocity profile of the particle at the outlet is obtained by averaging the individual particle velocity weighted by the intersection surfaces for each time step and for each calculation box area, given by $v_{k}=\sum_{i=1}^{N_{t}}\left(\sum_{j=1}^{N_{p}} s_{j}^{i} v_{j}^{i}\right) / \sum_{i=1}^{N_{t}}\left(\sum_{j=1}^{N_{p}} s_{j}^{i}\right)$, where $v_{j}^{i}$ represents the individual velocity of particle $j$ at time step $i, N_{t}$ represents the number of time steps to average, $N_{p}$ represents the number of particles intersecting with box $k$ at time step $i$, and $s_{j}^{i}$ is its intersection surface. The volume fraction is given by $\phi_{k}=\sum_{i=1}^{N_{t}}\left(\sum_{j=1}^{N_{p}} s_{j}^{i}\right) / \sum_{i=1}^{N_{t}} a d_{\max }$. 


\section{Continuum modeling}

Following Zhou et al. [6], we simulate the discharge flow of a granular medium from a rectangular silo considering a non-Newtonian incompressible Navier-Stokes system (equations are averaged across the thickness of the silo, leading to a pseudo-3D simulation in the spirit of Hele-Shaw flows):

$$
\begin{gathered}
\frac{\partial u_{i}^{p}}{\partial x_{i}}=0 \\
\rho\left[\frac{\partial u_{i}^{p}}{\partial t}+u_{j}^{p} \frac{\partial u_{i}^{p}}{\partial x_{j}}\right]=\frac{\partial \sigma_{i j}^{p}}{\partial x_{j}}+\rho g_{i}+f_{w i},
\end{gathered}
$$

where $u_{i}^{p}$ is the averaged velocity across the thickness $W$ of the cell and where $f_{w i}=-2 \frac{\mu_{w} p^{p}}{W} \frac{u_{i}^{p}}{\left|u_{i}^{p}\right|}$ represents Coulomb friction on the front and back walls (with a coefficient of friction $\left.\mu_{w}=0.1\right)$. The depth-averaged stress tensor of the particle phase $\sigma_{i j}^{p}=-p^{p} \delta_{i j}+\tau_{i j}^{p}$ comes only from direct particle-particle interactions and can be described by a sheardependant frictional rheology $[17,18]$ :

$$
\tau_{i j}^{p}=\eta_{p}\left(|\dot{\gamma}|, p^{p}\right) \dot{\gamma_{i j}} \quad \text { with } \quad \eta_{p}\left(|\dot{\gamma}|, p^{p}\right)=\frac{\mu(I) p^{p}}{|\dot{\gamma}|}
$$

with $I=|\dot{\gamma}| d / \sqrt{p^{p} / \rho_{p}}$, where $\dot{\gamma}_{i j}=\partial u_{i}^{p} / \partial x_{j}+\partial u_{j}^{p} / \partial x_{i}$ is the averaged strain-rate tensor and $|\dot{\gamma}|=\sqrt{\frac{1}{2} \dot{\gamma}_{i j} \dot{\gamma}_{i j}}$ its second invariant. For the shear-dependent friction coefficient, we take $\mu(I)=\mu_{s}+\Delta \mu /\left(I_{0} / I+1\right)$ with the constant $I_{0}=0.4$, $\mu_{1}=0.4$, and $\mu_{2}=0.68$ [19]. Note that this rheology is well adapted to the silo geometry $[6,9,20]$ but has several limits, such as compressibility problems [21], ill-posed problems [22], and tensorial formulation [23]. We have simulated an averaged two-dimensional (Hele-Shaw) silo of width $L$ and height $H=4 L$ with an inclined bottom at the angle $\theta_{i}$ towards the horizontal which ends up at a lateral orifice of dimension $D$ [see Fig. 2(b)]. The equations are made dimensionless using the silo width $L$ as a length scale, $\rho g L$ as a stress scale, and $\sqrt{L / g}$ as a timescale. The Navier-Stokes simulations are performed with the free software BASILISK [24], which uses a finite-volume projection method. Note that we use a regularization technique to avoid the divergence of the viscosity at low shear (for more details see [6]). The mesh is such that the width of the silo $L$ is divided into 64 computation cells (which is a good balance between precision and computational time).

Though a frictional boundary condition would be closer to the experimental setup behavior, we impose a no-slip condition at the solid boundaries. This is due to the penalization method used to solve the flow in complex geometries and has only little impact on the main results. We impose a zero pressure at the top and at the outlet. The dimensionless time step $\delta \tilde{t}=0.01$ is applied for the simulations. During the simulation, the volume of granular media left inside the silo is computed by following the granular surface every $\Delta \tilde{t}=0.1$, and the instantaneous mass flow rate of granular medium is determined using the variation of volume during $\Delta \tilde{t}=0.5$. In addition, the different fields (velocity and stress) are saved every $\Delta \tilde{t}=1$. We performed a series of simulations for the parameters given in Table II. The instantaneous flow rate being stationary during the discharge, we measure the mean
TABLE II. Geometrical parameters for the continuum simulations.

\begin{tabular}{lccc}
\hline \hline$D / L$ & $W / L$ & $\theta_{i}(\operatorname{deg})$ & $d_{p} / L$ \\
\hline$[0.44 ; 0.5 ; 0.56 ; 0.60 ;$ & {$[0.25 ; 0.5 ; 0.75 ;$} & {$[0 ; 10 ; 20 ; 30 ; 40 ;$} & $1 / 90$ \\
$0.63 ; 0.66 ; 0.69 ; 0.75]$ & $1.0 ; 2.0]$ & $50 ; 60 ; 70 ; 80]$ & \\
\hline \hline
\end{tabular}

flow rate $Q^{2 D}$ and we define the equivalent $3 \mathrm{D}$ flow rate as $Q=W Q^{2 D}$.

All the experimental and numerical data are available as Supplemental Material in a text file and the full continuum modeling simulation code used is available and discussed in the Supplemental Material [25].

\section{EFFECT OF THE BOTTOM INCLINATION FOR MONOLAYER FLOWS}

To study the influence of the bottom inclination angle on the silo discharge, we first focus on the simplest 2D case corresponding to the monolayer flow with the particles of diameter $d_{p}=4170 \mu \mathrm{m}$ in a silo of thickness $W=5 \mathrm{~mm}$. We compare the experimental results with those of the discrete 2D simulations, corresponding to an infinite thickness configuration without back and front sidewall effects. We first discuss the observation on the mass flow rate, and then we focus on the velocity fields and the flow properties at the center of the outlet which allow us to propose a simple model.

\section{A. Mass flow rate}

Figures 3(a) and 3(b) show the particle mass flow rate as a function of the inclination angle $\theta_{i}$ for various aperture sizes for (a) the experiments and (b) the 2D discrete simulations. In each case we observe that increasing the inclination of the bottom first tends to slightly increase the flow rate (until $\approx 40 \mathrm{deg}$ for the experiments and $\approx 20 \mathrm{deg}$ in the discrete simulations), and then we observe a strong decrease of the flow rate.

In a $2 \mathrm{D}$ flow, we expect the Hagen-Beverloo equation [Eq. (2)] to be valid. We have plotted in Figs. 3(c) and 3(d) the dimensionless mass flow rate $Q / \rho_{p} \phi_{b} \sqrt{g W^{5}}$ as a function of the dimensionless outlet size $D / W$ varying the bottom inclination for (c) the experiments and (d) the 2D discrete simulations. We observe that each curve can be effectively adjusted by Eq. (2), with a fitting parameter $c_{D}$ obtained using the least squares method, which depends on the bottom inclination $\theta_{i}$. To understand this behavior we can now analyze the velocity fields.

\section{B. Velocity fields}

The 2D experimental velocity fields of the granular flow at the front wall of the silo are shown in Fig. 4 for the experiments, with $D=40 \mathrm{~mm}$ for (a), (b) $\theta_{i}=0 \mathrm{deg}$ and (c), (d) $\theta_{i}=40$ deg. In Figs. 4(a) and 4(c) we have drawn the streamlines (calculated for velocities higher than $0.01 \mathrm{~m} / \mathrm{s}$ ). For a horizontal bottom, $\theta_{i}=0 \mathrm{deg}$, we observe a stagnant zone and a large slipping zone close to the outlet, whereas for $\theta_{i}=40 \mathrm{deg}$ all the particles are flowing and there is no stagnant zone anymore. In both cases the flow is vertical far 


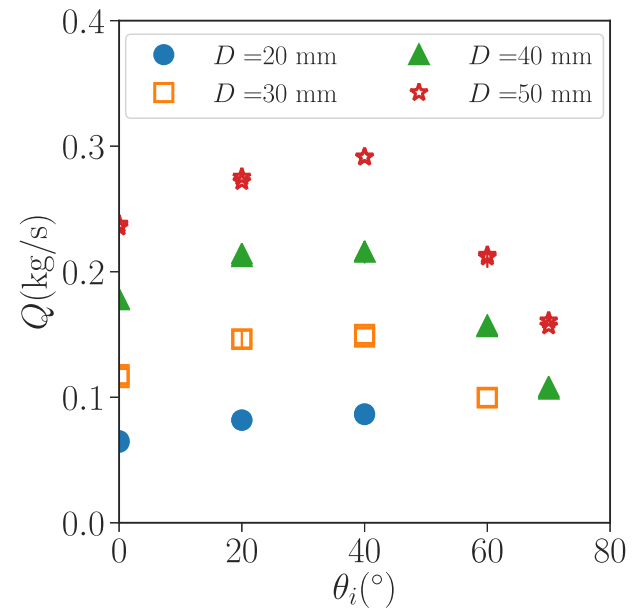

(a)

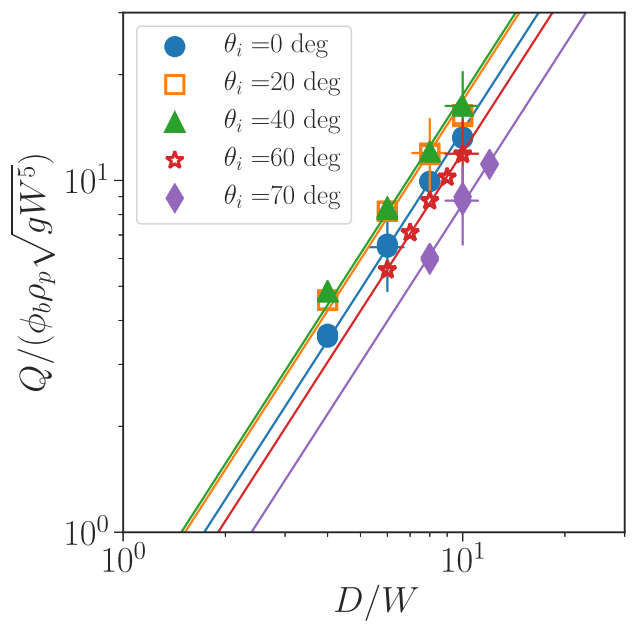

(c)

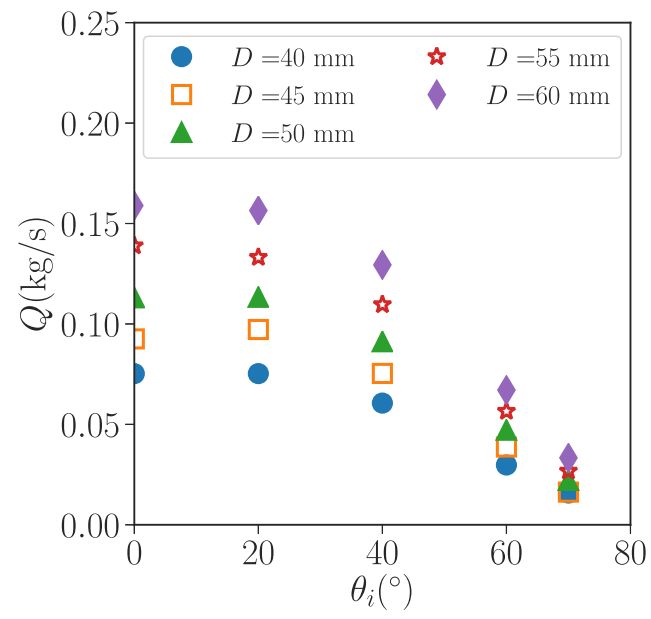

(b)

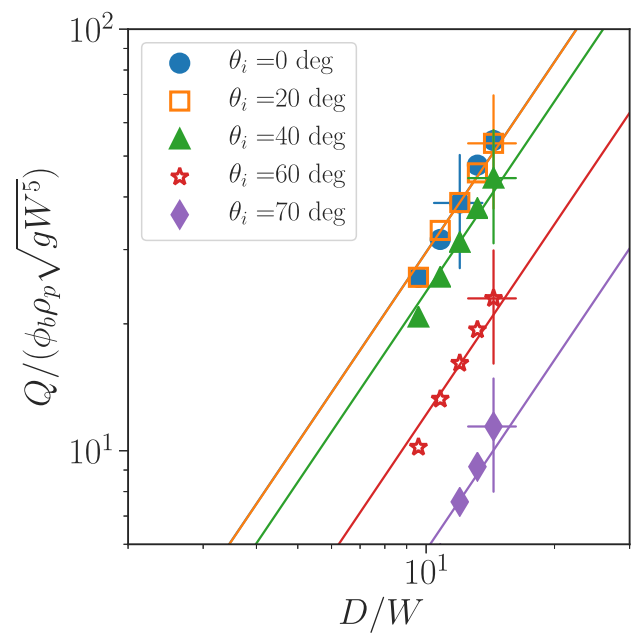

(d)

FIG. 3. Monolayer flow results for (a), (c) the experiments and (b), (d) the 2D discrete simulations. (a), (b) Mass flow rate of particles as a function of the bottom angle of inclination $\theta_{i}$ for several outlet sizes $D$ and (c), (d) mass flow rate of particles made dimensionless by $\phi_{b} \rho_{p} \sqrt{g W^{5}}$ vs the dimensionless outlet size $D / W$. The full lines represent the Hagen-Beverloo equation (2) with the fitting parameters $c_{D}$ which depend on $\theta_{i}$.

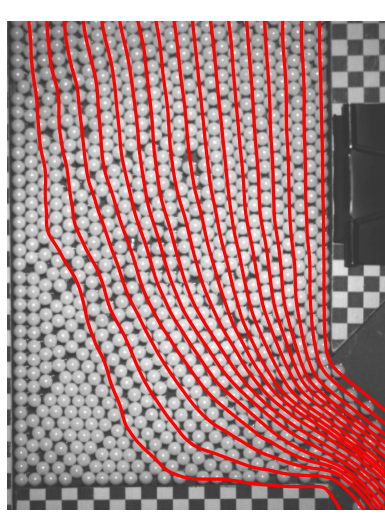

(a)

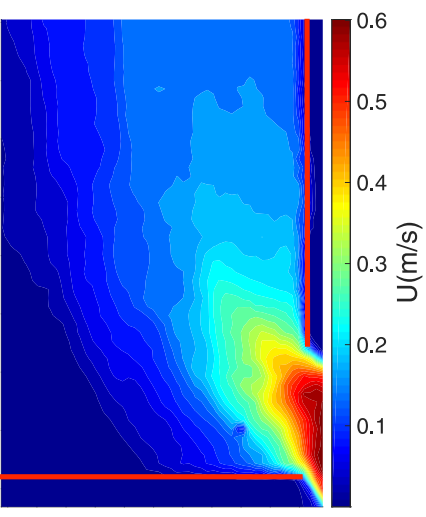

(b)

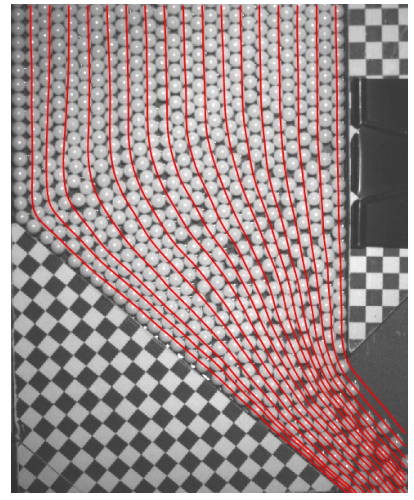

(c)

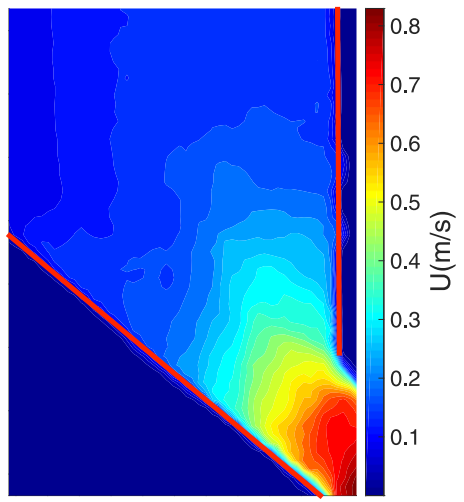

(d)

FIG. 4. (a), (c) Experimental streamlines and (b), (d) velocity magnitude fields for the monolayer experiments with $D=40 \mathrm{~mm}$ for (a), (b) $\theta_{i}=0 \mathrm{deg}$ and (c), (d) $\theta_{i}=40 \mathrm{deg}$. 


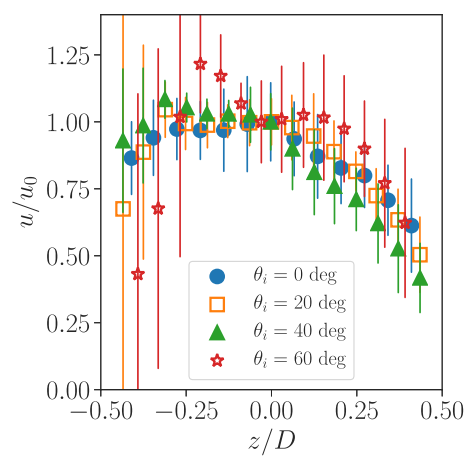

(a)

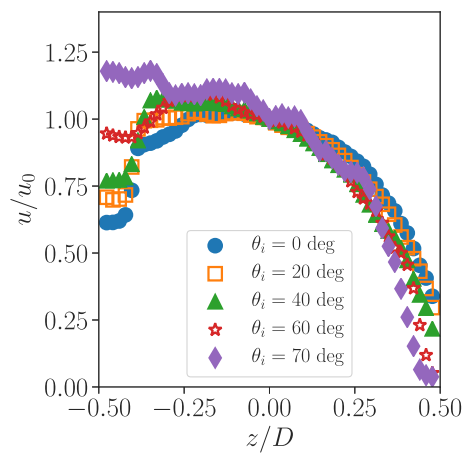

(d)

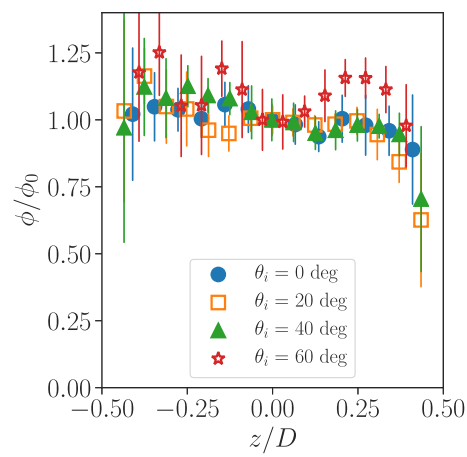

(b)

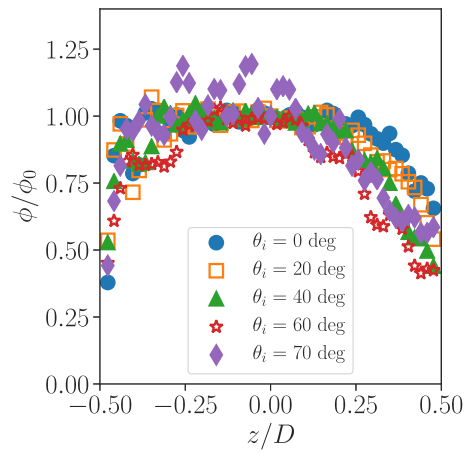

(e)

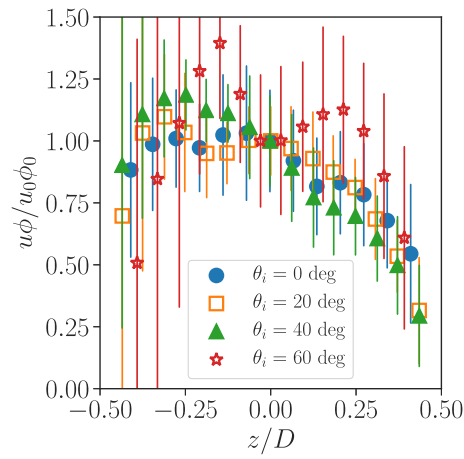

(c)

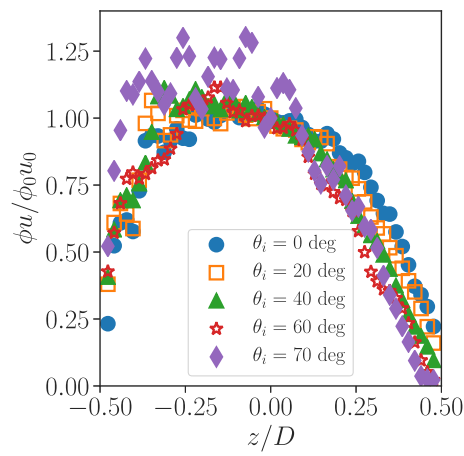

(f)

FIG. 5. Normalized experimental profiles at the outlet vs the normalized vertical position, $z / D$, for several bottom inclination $\theta_{i}$ for (a), (d) the horizontal velocity $u$, (b), (e) the volume fraction profiles $\phi$, and (c), (f) $\phi u$ for (a), (b), (c) the experiments with $D=40 \mathrm{~mm}$ and (d), (e), (f) the $2 \mathrm{D}$ discrete simulations with $D=50 \mathrm{~mm}$. The profiles are normalized with the corresponding value at the center of the outlet. The vertical position $z$ is oriented upward, and its origin is taken at the center of the outlet.

from the outlet, then rotates at the vicinity of the outlet. For an inclined bottom with $\theta_{i}=40 \mathrm{deg}$ the streamlines at the outlet appear to be quite aligned with the bottom.

The magnitude of the velocity in the front plane is illustrated by a color field in Figs. 4(b) and 4(d) for the same data. It clearly illustrates the flow behavior along the silo, with a zone of higher velocities at the very near region of the outlet where the particle acceleration is localized related to the flow cross-section reduction. We will then focus on the flow velocity at the outlet.

The outlet being vertical, the flow rate corresponds to the integral of the horizontal component of the velocity on the outlet area $Q=\rho_{p} \int_{-D / 2}^{D / 2} \int_{-W / 2}^{W / 2} \phi u d y d z$. Figures 5(a) and 5(d) show the profiles of the horizontal velocity component at the outlet, normalized by its value at the center of the outlet $u_{0}$ for a given outlet size $D$ and varying the angle of inclination of the bottom $\theta_{i}$ for (a) the monolayer experiments and (d) the $2 \mathrm{D}$ discrete simulations. We can first observe that the horizontal velocity profile exhibits an asymmetry between the top and the bottom of the outlet, certainly mainly due to the sliding of the particles on the inclined bottom. A difference between the experiments and the $2 \mathrm{D}$ discrete simulations is that in the latter case the sliding velocity increases with the bottom inclination. Except close to the bottom boundary, the dimensionless profiles are found to be rather self-similar, as observed experimentally by Janda et al. [4] for a bottom orifice or numerically in a $2 \mathrm{D}$ discrete simulation by Zhou et al. [6] for a lateral orifice. This self-similarity is observed whatever the inclination angle of the bottom and the outlet size.

The particle diameter not being negligible compared to the outlet size, the volume fraction cannot be simply evaluated using the bulk volume fraction $\phi_{b}$. Using the recorded images, we calculate the volume fraction, as can be seen in Fig. 6(a). We observe that the particles being monosize, they tend to crystallize far from the outlet. At the outlet this order is destroyed due to the converging flow, and as described in the literature [4-6], we observe that the particles tend to dilate, lowering the volume fraction. Figures 5(b) and 5(e) show the profile of the volume fraction across the outlet normalized by its value at the center of the outlet $\phi_{0}$ for a given outlet size $D$ and varying the angle of inclination of the bottom $\theta_{i}$ for (b) the monolayer experiments and (e) the 2D discrete simulations. We observe that the profiles are relatively flat and seem to be rather self-similar in both cases.

To predict the flow rate from the image processing, we then verify in Figs. 5(c) and 5(f) that the quantity $\phi u$, corresponding to the volumetric flux of particles transported, is also self-similar at the outlet varying the outlet size or the bottom inclination for both the experiments and the 2D discrete simulations. Then by averaging the profiles we evaluate $c=\int_{-D / 2}^{D / 2} \phi u /\left(\phi_{0} u_{0}\right) d z$, which is found to be rather constant with $c_{0}=\langle c\rangle=0.89$ for the experiments and $c_{0}=\langle c\rangle=$ 0.78 for the $2 \mathrm{D}$ discrete simulations. Finally, Figs. $6(\mathrm{~b})$ and 


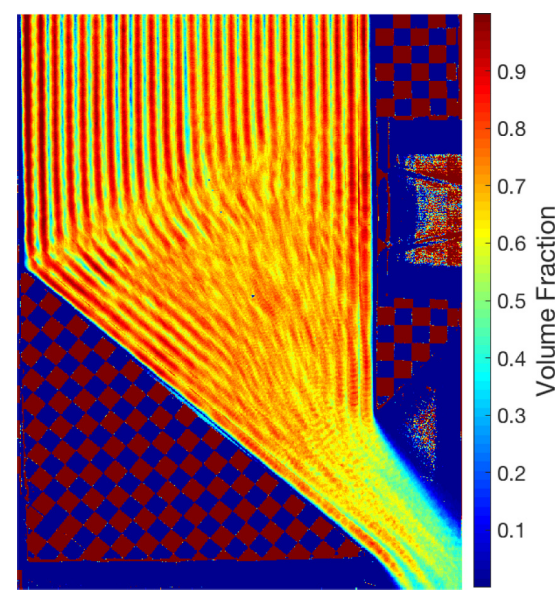

(a)

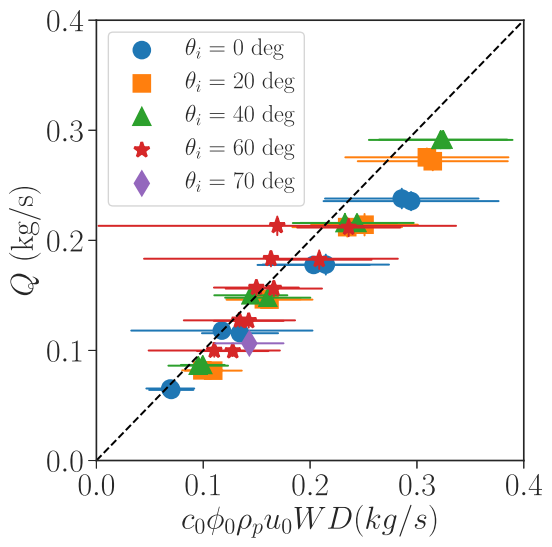

(b)

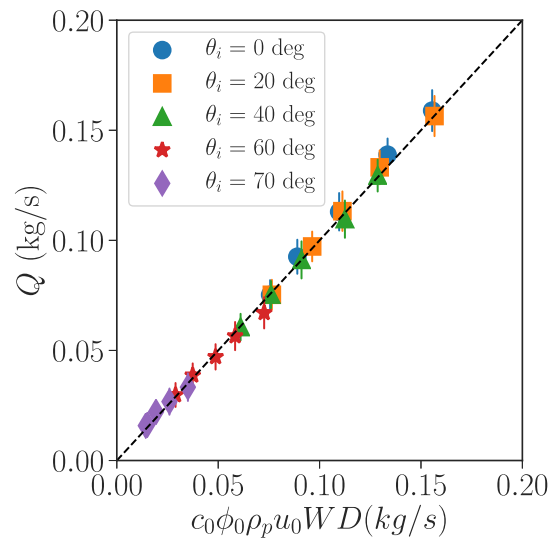

(c)

FIG. 6. (a) Map of the volume fraction for $\theta_{i}=40 \mathrm{deg}$ and $D=40 \mathrm{~mm}$. (b), (c) Flow rate vs $c_{0} \phi_{0} \rho_{p} u_{0} W D$ for (a) the monolayer experiments with $c_{0}=0.89$ and (b) the $2 \mathrm{D}$ discrete simulations with $c_{0}=0.78$. The dashed line represents Eq. (8).

6(c) show that the flow rate is reasonably given in both cases by

$$
Q=c_{0} \rho_{p} \phi_{0} u_{0} W D
$$

The flow rate being controlled by the horizontal component of the velocity $u_{0}$ and the volume fraction $\phi_{0}$ at the center of the outlet, let us now consider how these variables depend on the control parameters of the experiments or the $2 \mathrm{D}$ simulations.

\section{Flow properties at the center of the outlet: Towards a simple model}

We first focus on the volume fraction at the center of the outlet $\phi_{0}$. Following [4-6] we can expect that the dilation at the outlet is due to a geometrical constraint, the particle diameter not being negligible compare to the outlet size. For a bottom orifice or a lateral orifice, those papers have shown that the volume fraction at the outlet depends mainly on the number of beads in the aperture $D / d_{p}$, and is well described by $\phi_{0}=\phi_{b} G\left(D / d_{p}\right)$ where $G(x)=\xi_{\phi}\left(1-\alpha e^{-\beta x}\right)$. However, in our case, the bottom being inclined, the smaller dimension in the silo is not the outlet $D$ but the perpendicular at the bottom which joins the top of the outlet, whose dimension is $D \cos \theta_{i}$. In Fig. 7 we have plotted $\phi_{0} / \phi_{b}$ versus $D \cos \theta_{i} / d_{p}$ for both (a) the experiments and (b) the 2D discrete simulations. We find that the data seem to superimpose and can be reasonably adjusted by

$$
\phi_{0}=\phi_{b} \xi_{\phi}\left(1-\alpha e^{-\beta D \cos \theta_{i} / d_{p}}\right)
$$

with $\xi_{\phi}=0.9, \alpha=0.6$, and $\beta=0.3$ for the monolayer experiments and $\xi_{\phi}=0.95, \alpha=1.42$, and $\beta=0.33$ for the $2 \mathrm{D}$ discrete simulations, in good agreement with previous work [6]. We can, however, notice that the variation of $\phi_{0} / \phi_{b}$ stays small in the range explored and is a second order effect on the variation of the flow rate with the inclination angle of the bottom.

We now turn to the horizontal component of the velocity at the center of the outlet. Following Zhou et al. [6], we characterize it using the magnitude of the velocity $U_{0}$ and its inclination $\theta_{0}$ toward the horizontal with

$$
u_{0}=U_{0} \cos \theta_{0}
$$

We can first focus on the role of the inclination of the bottom of the silo $\theta_{i}$ on the inclination of the flow at the central streamline $\theta_{0}$, as illustrated in Fig. 8. In Fig. 8(a) we observe that for a given bottom inclination the central streamline inclination does not depend on the outlet size $D$. In Fig. 8(b) we have plotted the mean value of $\cos \theta_{0}$ [corresponding to the dashed horizontal lines in Fig. 8(a)] versus $\cos \theta_{i}$. We observe that except for the horizontal bottom $\left(\theta_{i}=0\right)$, the inclination of the flow is strongly correlated to the bottom inclination, the cosine of both angles being well adjusted by a linear function, $\cos \theta_{0}=\zeta \cos \theta_{i}$, with $\zeta=0.92$ (see the dashed line in the figure). This correlation may be explained by the profile of the streamline inclination at the outlet, which is found to be quite linear between the bottom inclination and a vertical inclination at the top (data not shown). For small bottom inclinations, the central streamline seems to reach a constant inclination that we denote $\theta_{0}^{f} \approx 36 \mathrm{deg}$. This regime

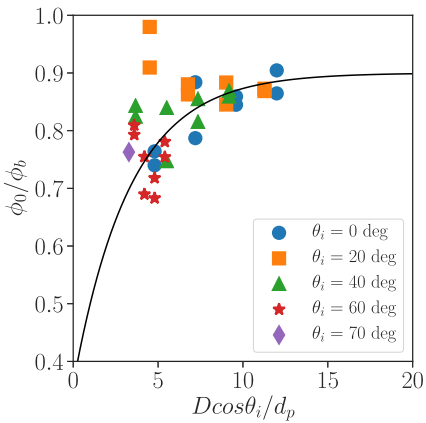

(a)

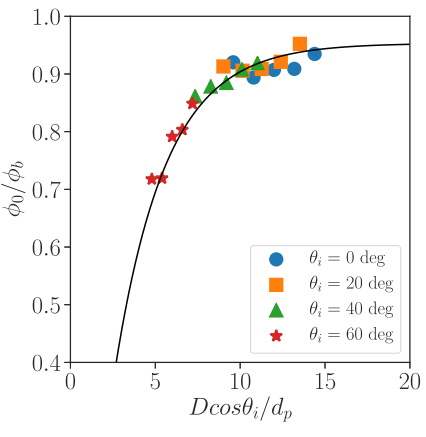

(b)
FIG. 7. Volume fraction at the center of the outlet $\phi_{0}$ normalized by the bulk volume fraction $\phi_{b}$ vs the normalized outlet size, $D \cos \theta_{i} / d_{p}$, for various bottom inclinations for (a) the experiments and (b) the $2 \mathrm{D}$ discrete simulations. The black line represents Eq. (9), with (a) $\xi_{\phi}=0.9, \alpha=0.6$, and $\beta=0.3$, and (b) $\xi_{\phi}=0.95$, $\alpha=1.42$, and $\beta=0.33$. 


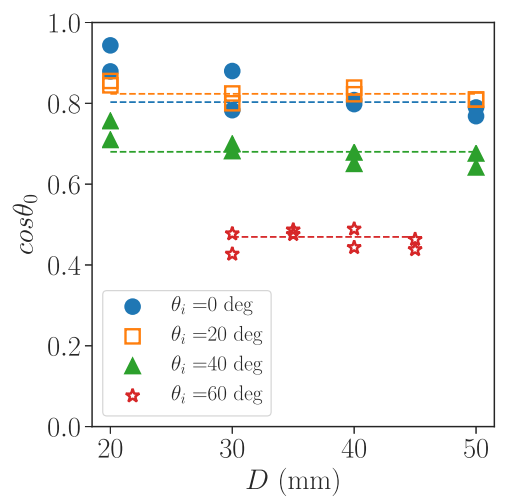

(a)

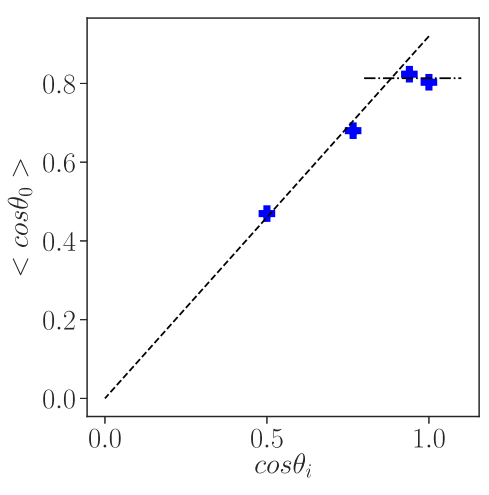

(b)

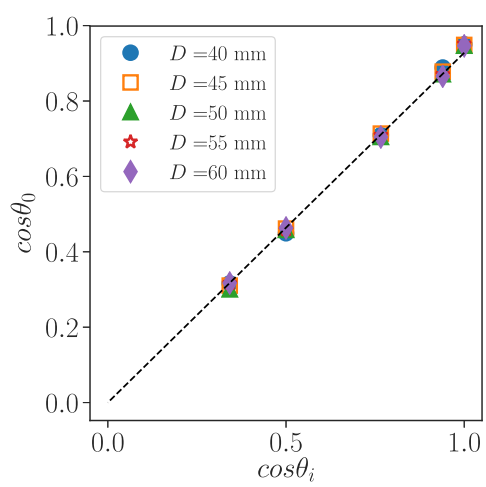

(c)

FIG. 8. (a) Experiments: Inclination of the central streamline at the outlet, $\cos \theta_{0}$, vs the outlet size $D$ for various inclinations of the bottom of the silo. The dashed lines represent the mean value plotted in (b) vs $\cos \theta_{i}$ where the dashed black line represents Eq. (12) with $\zeta=0.92$ and the dashed dotted line represents $\cos \theta_{0}^{f}=0.81$. (c) 2D discrete simulations: Inclination of the central streamline at the outlet, $\cos \theta_{0}$ vs $\cos \theta_{i}$. The dashed black line represents Eq. (12) with $\zeta=0.93$.

of constant inclination may be due to the presence of an angle of avalanche. If we suppose that the flow is unperturbed by the bottom while the bottom inclination is smaller than the streamline angle generated by a horizontal bottom, i.e., $\theta_{0}<\theta_{0}^{f}$, we obtain a model for the streamline angle at the center of the outlet:

$$
\begin{aligned}
& \text { if } \theta_{i}<\theta_{c}, \quad \cos \theta_{0}=\cos \theta_{0}^{f}, \\
& \text { else } \quad \cos \theta_{0}=\zeta \cos \theta_{i} .
\end{aligned}
$$

We denoted $\theta_{c}=\arccos \left(\cos \theta_{0}^{f} / \zeta\right) \approx 28 \mathrm{deg}$ the critical angle at which the transition occurs. This model suggests that when the bottom inclination increases above a threshold $\theta_{c}$, the central streamline inclination is controlled by the bottom inclination and not anymore by the internal friction. Thus the flow rate will tend to decrease, the flow being less horizontal. Concerning the 2D discrete simulations, when the bottom is horizontal we measure $\theta_{0}^{f}=18.5 \mathrm{deg}$, a value that is close to the angle of friction $\delta=\operatorname{atan}\left(\mu_{p}\right)=21.8 \mathrm{deg}$. Then we can see in Fig. 8(c) that, similarly to the experimental case, the inclination of the flow is well described by Eq. (12) with $\zeta=0.93$. However, we observe that $\cos \theta_{0}^{f} / \zeta>1$. Thus we will consider that there is no plateau regime in the simulation corresponding to $\theta_{c}=0$.

We now turn to the magnitude of the velocity. For the experiments, in Fig. 9(a) the normalized magnitude of the velocity of the central streamline is plotted versus the normalized orifice size for various bottom inclinations. As observed numerically by Zhou et al. [6], the velocity magnitude is well adjusted by a square root variation with respect to the opening size given by Eq. (3). However, the fitting parameter appears to depend strongly on the bottom inclination in this new configuration. The scaling law suggests that velocity magnitude $U_{0}$ is controlled by a transfer from the potential energy to kinetic energy in the characteristic length of the outlet size. However, when the flow orientation is controlled by the bottom inclination, we can suppose that the flow is not free to rotate and the inclination at the central streamline is given by Eq. (12). When reaching the dense granular zone, at a distance $s \sim D$, the potential energy then depends on the orientation of the streamline, $E_{p} \sim \rho_{p} g \sin \theta_{0} s$. This potential energy is transferred to kinetic energy at the outlet $E_{k} \sim \rho_{p} U_{0}^{2}$, thus giving

$$
U_{0}=c_{E} \sqrt{g D \sin \theta_{0}},
$$

which predicts an increase of the velocity with the bottom inclination, as seen in Fig. 9(a). In Fig. 9(b) we have plotted the normalized velocity $U_{0} / \sqrt{g L}$ vs $D \sin \theta_{0} / L$ for all the data, and we observe a good collapse. The data are well adjusted by Eq. (13) with $c_{E}=1.29$. This effect tends to slightly counterbalance the effect of the inclination of the streamline on the flow rate. The main difference between the $2 \mathrm{D}$ discrete simulation and the monolayer experiments lies in the magnitude of the velocity on the central streamline at the outlet. Indeed, in Fig. 9(c) we can see that $U_{0}$ does not sensitively depend on $\theta_{i}$. The data can be adjusted by Eq. (3) with $c_{E}=1.45$. This difference may be linked to either the fact that the monolayer flow is not a true 2D flow but encounters friction at the front and rear wall, or to the boundary condition at the bottom wall: we observe stronger sliding in the simulation than in the experiments. New 2D discrete simulations testing these hypotheses by simulating a true monolayer flow with back and front sidewalls and by varying the wall friction coefficient should answer this question.

From all the previous analysis, it is possible to deduce a model for the flow-rate dependence on the bottom inclination of the silo. Using Eqs. (8)-(13) we can write

$$
\begin{gathered}
\text { if } \theta_{i}<\theta_{c}: \quad \tilde{Q}=c_{D} \\
\text { else: } \quad \tilde{Q}=c_{\theta} \cos \theta_{i}\left(1-\zeta^{2} \cos ^{2} \theta_{i}\right)^{1 / 4}
\end{gathered}
$$

where $\quad \tilde{Q}=Q /\left[\rho_{p} \phi_{b} G\left(D \cos \theta_{i} / d_{p}\right) D W \sqrt{g D}\right]$, with $G(x)=\xi_{\phi}\left(1-\alpha e^{-\beta x}\right), \theta_{c}=\arccos \left(\cos \theta_{0}^{f} / \zeta\right), c_{\theta}=c_{0} c_{E} \zeta$, and $c_{D}=c_{0} c_{E} \cos \theta_{0}^{f} \sqrt{\sin \theta_{0}^{f}}$. For large bottom inclination and large outlet size, Eq. (15) can be simplified to give Eq. (16), with less than $15 \%$ of error for $\theta_{i}>40^{\circ}$, which also 


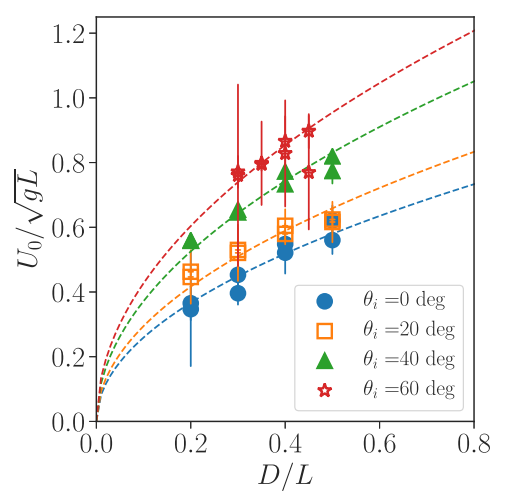

(a)

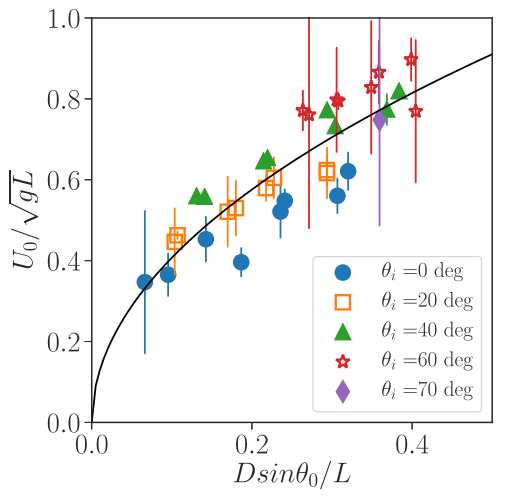

(b)

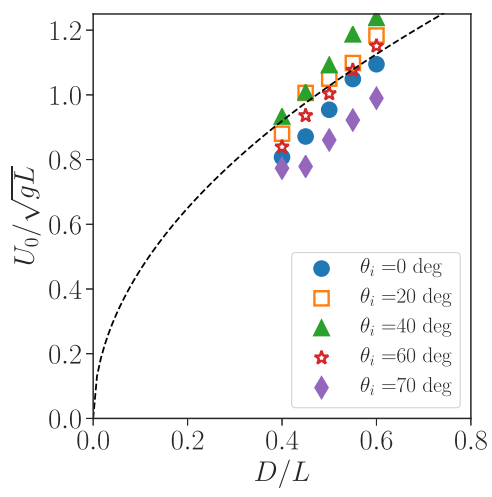

(c)

FIG. 9. Magnitude of the experimental velocity profiles at the center of the outlet $U_{0}$ normalized by $\sqrt{g L}$ vs the outlet size $D$ normalized by (a), (c) $L$ and (b) $L / \sin \theta_{0}$ for several outlet inclinations $\theta_{i}$ for (a), (b) the experiments and (c) the 2D discrete simulations. The dashed lines represent Eq. (3) with (a) adjustable coefficients which depends on $\theta_{i}$ and (c) $c_{E}=1.45$ obtained by the least squares method. The full line represents Eq. (13) with $c_{E}=1.29$.

corresponds to the 2D discrete simulations case:

$$
\tilde{Q}=c_{\theta} \cos \theta_{i} \text {. }
$$

In Fig. 10 we compare the normalized flow rate versus the angle of inclination with the model given by Eqs. (14) and (15) (full line), and Eq. (16) (dashed line). The agreement is fairly good, except for the experiment's low bottom inclination (below $\theta_{c}$ ), where the model predicts a plateau and not a slight increase with $\theta_{i}$. This may be due to a large sliding zone at the bottom for low angles, as can be seen in Figs. 4(a) and 4(b). Even though this simple model does not finely capture this particular behavior, it is able to predict the main physical mechanism of the influence of the bottom inclination on the flow rate for a $2 \mathrm{D}$ flow. The $2 \mathrm{D}$ discrete simulations reproduce most of the behavior observed, except for the variation of $U_{0}$ with $\theta_{i}$. For future studies it may be an interesting tool to test the effect of the front and back sidewalls, to see the effect of the grain and wall parameters $\left(\mu_{p}\right.$ and $\left.\mu_{w}\right)$ on the flow rate and to obtain information on the stress fields.

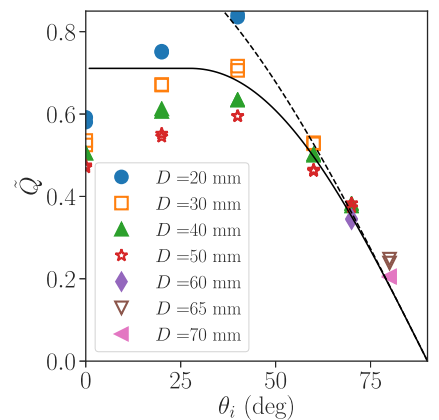

(a)

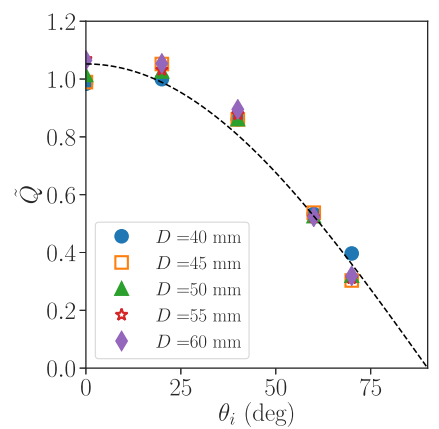

(b)
FIG. 10. Experimental mass flow rate normalized by $\rho_{p} \phi_{b} G\left(\frac{D \cos \theta_{i}}{d_{p}}\right) D W \sqrt{g D}$ vs the bottom angle of inclination for (a) the experiments and (b) the $2 \mathrm{D}$ discrete simulations. The full line represents Eqs. (14) and (15), and the dashed lines represent Eq. (16) with (a) $\xi_{\phi}=0.9, \alpha=0.6, \beta=0.3, \zeta=0.92, c_{E}=1.29$, $c_{0}=0.89, \quad \theta_{c}=28^{\circ}$, and $\cos \theta_{0}^{f}=0.81$, and (b) $\xi_{\phi}=0.95$, $\alpha=1.42, \beta=0.33, \zeta=0.93, c_{E}=1.45$, and $c_{0}=0.78$.
In the next section we will study 3D effects with finite size of the silo and explore how this configuration can change the observed behaviors.

\section{ROLE OF THE BOTTOM INCLINATION FOR 3D FLOWS}

To study the role of the bottom inclination for 3D flows we first focus on the experiments performed with one of the smallest particle sizes having the velocity fields resolved, $d_{p}=538 \mu \mathrm{m}$. We compare the experimental results with those of the continuum simulations and follow the same approach as for the $2 \mathrm{D}$ flow.

\section{A. Mass flow rate}

Figures 11(a) and 11(b) show the particle mass flow rate as a function of the inclination angle $\theta_{i}$ for a given aperture size and various silo thicknesses for (a) the experiments and (b) the continuum simulations. In these 3D flows we observe in both cases a large plateau where the mass flow rate does not depend on the inclination of the bottom, much larger than for the 2D case. For example, in the experiments for a narrow silo of thickness $W=5 \mathrm{~mm}$ the inclination of the bottom does not influence the flow for angles as large as $60 \mathrm{deg}$. In this low- $\theta_{i}$ regime we do not observe the same increase as for the $2 \mathrm{D}$ configuration. Then above a critical angle, which seems to depend on the silo thickness, the mass flow rate starts to decrease. The flow rate of the plateau can be evaluated by the value at $\theta_{i}=0$, which corresponds to a rectangular silo with a lateral hole as studied by Zhou et al. [6]. Following this work, we have plotted in Figs. 11(c) and 11 (d) the dimensionless mass flow rate $Q /\left(\rho_{p} \phi_{b} \sqrt{g W^{5}}\right)$ as a function of the aspect ratio $\mathcal{A}=D / W$ for several inclinations of the bottom of the silo. As predicted by Zhou et al. [6], for the horizontal silo $\left(\theta_{i}=0 \mathrm{deg}\right)$ the flow rate can be adjusted by Eq. (4) (see the black lines) with respectively $c_{D}=0.5$ and $\gamma=0.44$ for the experiments and $c_{D}=0.8$ and $\gamma=0.35$ for the continuum simulations, in good agreement with the 


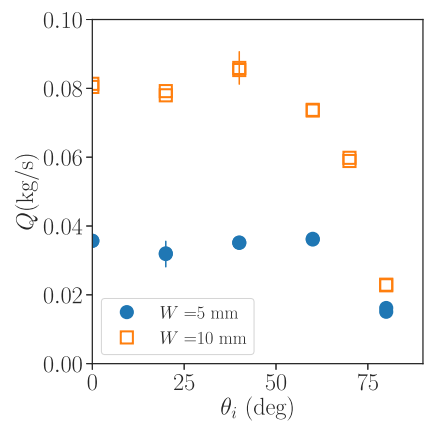

(a)

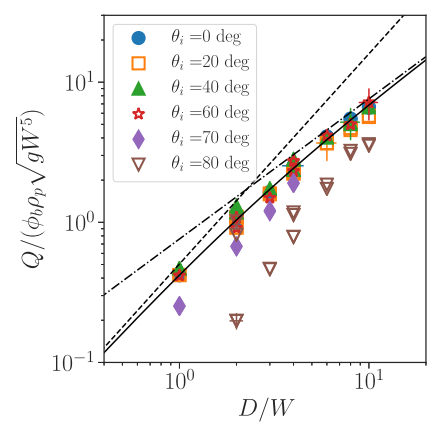

(c)

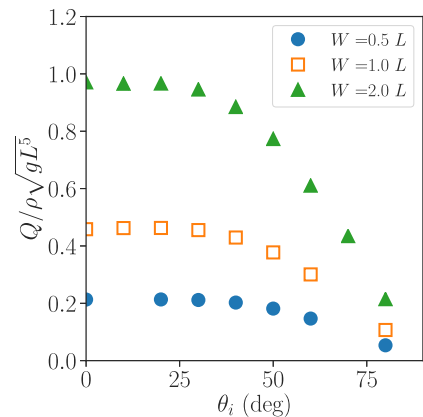

(b)

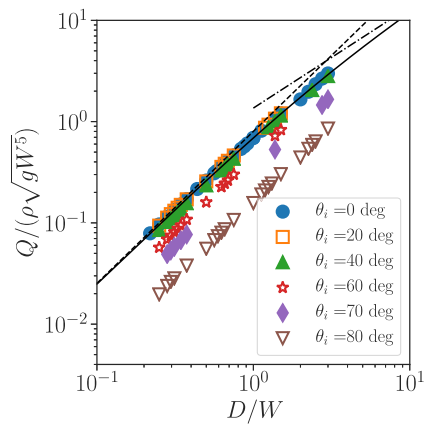

(d)
FIG. 11. 3D flow results for (a), (c) the experiments with $d_{p}=538 \mu \mathrm{m}$ and (b), (d) the continuum simulations. (a), (b) Mass flow rate of particles as a function of the bottom angle of inclination $\theta_{i}$ for several silo thicknesses $W$ with (a) $D=30 \mathrm{~mm}$ and (b) $D=0.75 L$. (c), (d) Mass flow rate of particles made dimensionless by $\phi_{b} \rho_{p} \sqrt{g W^{5}}$ vs the dimensionless outlet size $D / W$ for several bottom inclinations $\theta_{i}$ explored. The solid line represents Eq. (4) with (c) $c_{D}=0.5$ and $\gamma=0.44$ and (d) $c_{D}=0.8$ and $\gamma=0.35$. The dashed line represents the Hagen-Beverloo regime [Eq. (2)] and the dashed-dotted line represents the linear asymptotic regime of Eq. (4) for $D / W \gg 1$.

values reported in Ref. [6]. We thus recover that the granular flow can be separated into two regimes. For a small aperture aspect ratio $D / W \ll 1$, on the left part of Figs. 11(c) and 11(d) the data can be adjusted by the Hagen-Beverloo law given by Eq. (2) corresponding to a flow controlled by the internal friction. The second regime of granular flow is observed for a large aperture aspect ratio $D / W \gg 1$. Indeed, on the right part of Figs. 11(c) and 11(d) the results can be adjusted by a linear law, corresponding to a flow controlled by the wall friction (see the dashed-dotted lines). The transition between these two regimes occurs at around $D / W \approx 2$. The experiments were mainly done in the transition zone between the two regimes, whereas the continuum simulations were mainly performed in the Hagen-Beverloo regime and at the beginning of the transition zone, which gives imprecision on the determination of the coefficient $\gamma$. When we increase the bottom inclination, we observe that for a large range of inclination angles the data are superimposed with those of the horizontal inclination; then we observe a departure which seems to begin for the smaller aperture aspect ratio. In the following part, we discuss the velocity fields.

\section{B. Velocity fields}

The 2D velocity fields of the granular flow at the front wall of the silo are shown in Figs. 12(a) and 12(b) for the experiments with the thinner silo, $W=5 \mathrm{~mm}, D=40 \mathrm{~mm}, \theta_{i}=40$ deg, and $d_{p}=538 \mu \mathrm{m}$, and for Figs. 12(c) and 12(d) the continuum simulations with $W=2 L, D=0.75 L$, and three bottom inclinations. In Figs. 12(a) and 12(c) we have drawn the streamlines, and we can see that the flow is quasivertical far from the outlet, and then it rotates in the vicinity of the outlet. Note in Fig. 12(a) that under the conditions of the experiment, the inclination of the streamlines at the outlet appears to be higher than the inclination of the bottom of the silo. We can also observe a large stagnant zone located on the left side of the silo. In the continuum simulations, we observe that for a small inclination $\left(\theta_{i}=20 \mathrm{deg}\right)$ the inclination of the streamlines is not perturbed compared to the horizontal bottom $\left(\theta_{i}=0 \mathrm{deg}\right)$ except close to the inclined bottom, leading to a reduce stagnant zone. For large inclination $\left(\theta_{i}=60 \mathrm{deg}\right)$ the streamlines inclinations seem to be controlled by the bottom inclination, and the stagnant zone connects to the inclined bottom largely upward from the outlet and tend to disappear.

Figures 12(b) and 12(d) show the magnitude of the velocity in the front plane for the same data. Again, it illustrates the flow behavior along the silo. A stagnant zone lies on the left side, which is quite large in the experiment although we imposed a significant angle of inclination. A zone of higher velocities is observed at the very near region of the outlet where the particle acceleration is localized. This may again explain the plateau observed for the mass flow rate when varying the angle of inclination (Fig. 11). In the zero inclination bottom case, with the lateral aperture the stagnant zone is confined in the left-hand side, bottom part of the silo, its boundary having a given inclination with respect to the bottom of the silo. The small inclination of the bottom only suppresses particles within this zone, which would have no interaction with the flow. It therefore does not impact the flow rate. For larger bottom inclination, the flowing zone is constrained by the silo geometry, which affects the flow orientation and decreases the discharge flow rate. To test this hypothesis we will focus again on the flow velocity at the center of the outlet, but first we verify that the image processing at the front wall is successful in predicting the flow rate in the $3 \mathrm{D}$ case. The particles are small enough compared to the outlet dimensions ( $D \cos \theta_{i} / d_{p}>10$ ) to suppose the volume fraction at the outlet constant $\phi \approx \phi_{b}$ [see Fig. 7(a)]; then if the profile of the horizontal velocity is self-similar, the flow rate should read

$$
Q=c_{0} \rho_{p} \phi_{b} u_{0} W D \text {. }
$$

Figure 13 shows the profiles of the horizontal velocity component at the outlet, normalized by its value at the center of the outlet $u_{0}$ for (a), (b) the experiments with $d_{p}=$ $538 \mu \mathrm{m}$ and (d), (e) the continuum simulations, varying (a), (d) the angle of inclination of the bottom and (b), (e) the thickness of the silo for each graph, the other parameters being kept constant. We observe that these profiles are rather self-similar when varying all the parameters in both cases. Indeed, the integral of the normalized profile $c=\int_{-D / 2}^{D / 2} u / u_{0} d z$ is found to be rather constant, with $c_{0}=\langle c\rangle=0.79$ for 


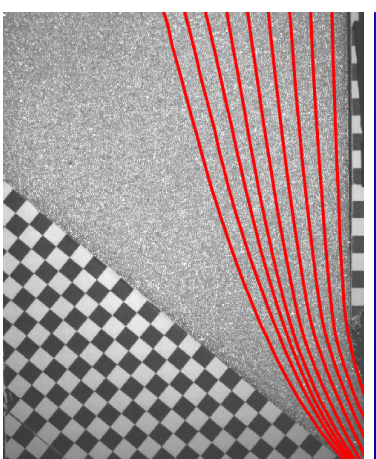

(a)

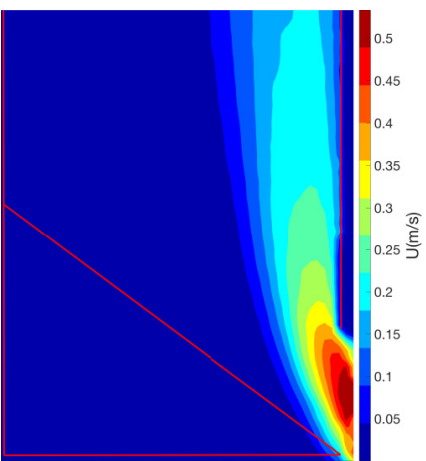

(b)

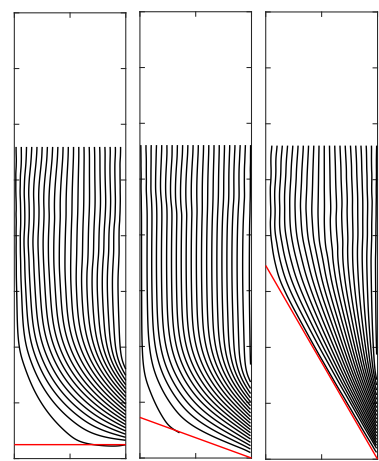

(c)
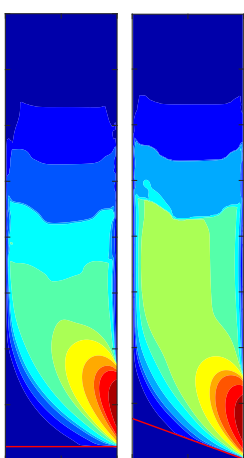

(d)

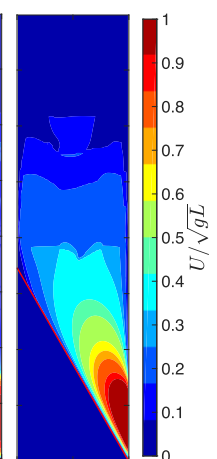

FIG. 12. (a), (c) Streamlines and (b), (d) velocity magnitude fields for (a), (b) the 3D experiments with $W=5 \mathrm{~mm}, d_{p}=538 \mathrm{~mm}$, $D=40 \mathrm{~mm}$, and $\theta_{i}=40 \mathrm{deg}$, and (c), (d) the continuum simulation with $W=2 L, D=0.75 L$ and from left to right $\theta_{i}=0$ deg, 20 deg, and $60 \mathrm{deg}$.

the experiments and $c_{0}=\langle c\rangle=0.81$ for the continuum simulation (data not shown). We can also observe that the horizontal velocity profile exhibits an asymmetry between the top and the bottom of the outlet but smaller than in the 2D case (see Fig. 5). Figure 13(c) shows the comparison between the experimental flow rate obtained from the balance

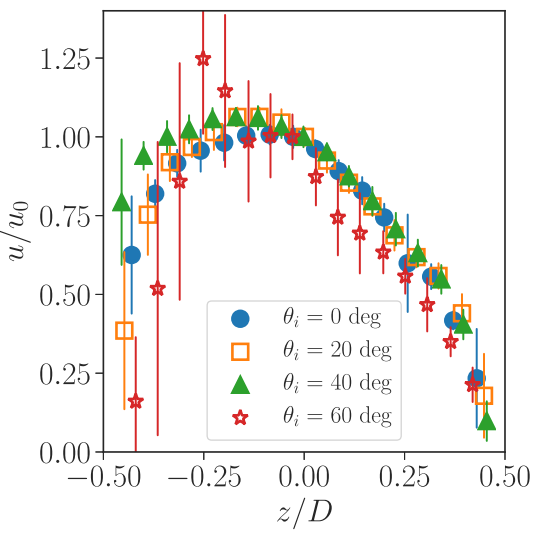

(a)

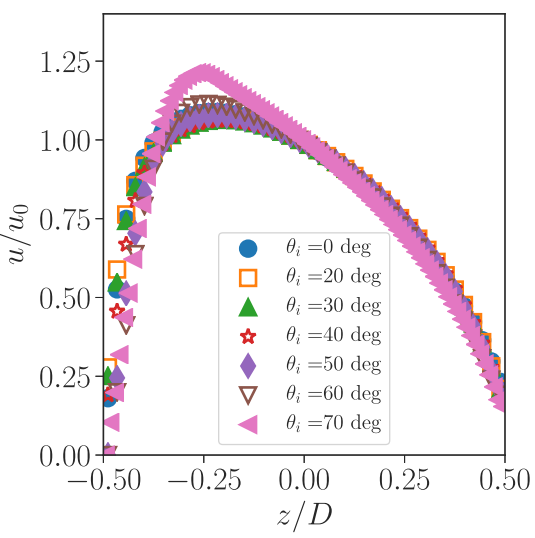

(d)

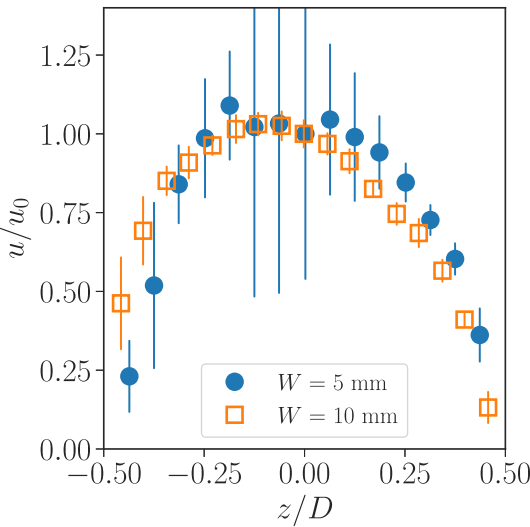

(b)

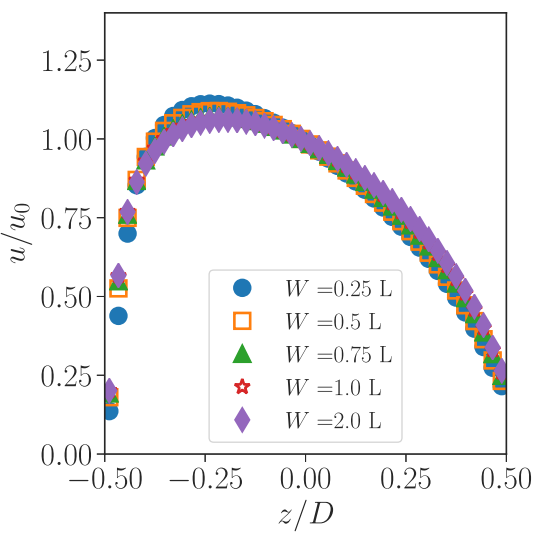

(e)

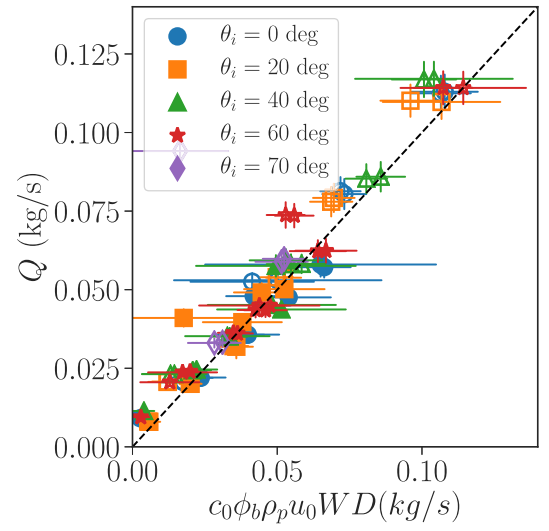

(c)

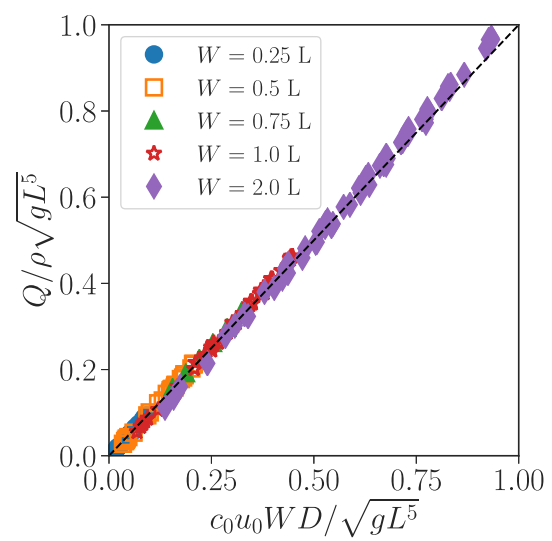

(f)

FIG. 13. (a), (b), (d), (e) Horizontal velocity profiles at the outlet, $u$, normalized by the velocity at the center, $u_{0}$, vs the vertical position normalized by the outlet size $z / D$ for (a), (b) the experiments with $d_{p}=538 \mu \mathrm{m}$ and (a) $D=40 \mathrm{~mm}, W=10 \mathrm{~mm}$ and several bottom inclinations $\theta_{i}$, (b) $\theta_{i}=0 \mathrm{deg}, D=40 \mathrm{~mm}$, and several silo thicknesses $W$, and for (d), (e) the continuum simulations with (d) $D=0.68 L$, $W=0.5 L$ and several outlet inclinations $\theta_{i}$, and (e) $\theta_{i}=0 \mathrm{deg}, D=0.68 L$ and several silo thicknesses $W$. (c), (f) Flow rate vs $c_{0} \phi_{b} \rho_{p} u_{0} W D$ for (c) the experiments with $d_{p}=538 \mu \mathrm{m}, W=5 \mathrm{~mm}$ (closed symbols), and $W=10 \mathrm{~mm}$ (opened symbols), and (f) the continuum simulations in a dimensionless representation. The dashed lines represent the equation $y=x$ with (c) $c_{0}=0.79$ and (f) $c_{0}=0.81$. 


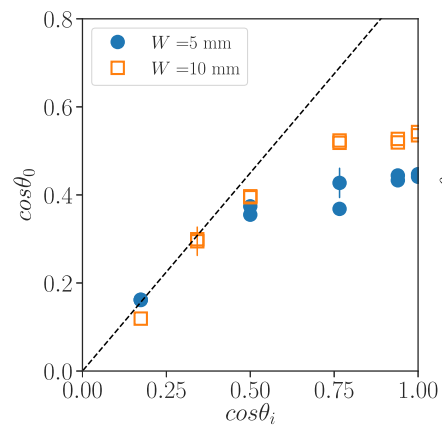

(a)

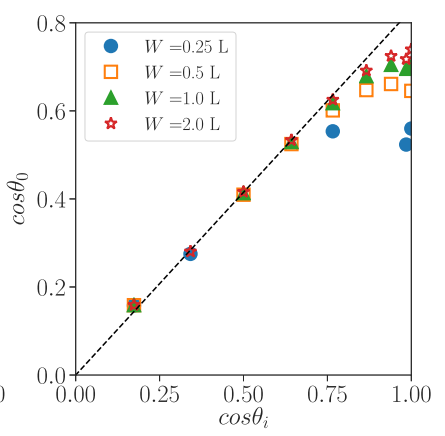

(b)
FIG. 14. Inclination of the central streamline at the outlet, $\cos \theta_{0}$, vs the inclination of the bottom of the silo, $\cos \theta_{i}$, for (a) the experiments with $d_{p}=538 \mu \mathrm{m}$ and $D=30 \mathrm{~mm}$ and (b) the continuum simulations with $D=0.75 \mathrm{~L}$. The dashed lines represent Eq. (12) with (a) $\zeta=0.9$ and (b) $\zeta=0.83$.

measurement and Eq. (17) obtained from the image processing. We can see that the data obtained by the image processing are scattered (in particular when $\theta_{i}$ increases) but the agreement is fairly good, with a small discrepancy for the larger silo (opened symbols). This may be due to $3 \mathrm{D}$ effects, which would tend to reduce the velocity at the front wall, leading to Eq. (17) slightly underestimating the flow rate. Finally, Fig. 13(e) shows that Eq. (17) is also valid for the continuum simulation.

We can now turn to the study of the flow properties at the center of the outlet, and following the previous part, we will focus on the magnitude of the velocity $U_{0}$ and its inclination $\theta_{0}$, with $u_{0}=U_{0} \cos \theta_{0}$.

\section{Flow properties at the center of the outlet: Generalization of the simple model}

The variation of the inclination of the central streamline with the bottom inclination is shown in Fig. 14 for a given outlet size and various silo thicknesses, for both the experiments and the continuum simulations. Similar to the monolayer experiments, we recover that for large bottom inclinations (small $\cos \theta_{i}$ ) the inclination of the flow is strongly correlated to the bottom inclination, following Eq. (12) with $\zeta=0.9$ (resp. 0.83 ) for the experiments (resp. the continuum simulations). For low bottom inclinations, the flow is not perturbed by the inclined bottom and $\cos \theta_{0}$ tends to a plateau, which depends on the silo thickness $W$. We denote $\theta_{0}^{f}$ the angle of inclination of the central streamline at the outlet on the plateau, its value being defined for the horizontal bottom case $\left[\cos \left(\theta_{i}\right)=1\right]$. In Fig. $15 \cos \theta_{0}$ is plotted versus the outlet aspect ratio $D / W$. As predicted by Zhou et al. [6], we observe that for $\theta_{i}=0$ the inclination clearly scales with $D / W$ and is well adjusted by Eq. (5) (see the black line) with $c_{\theta_{0}}=0.78$ and $\gamma=0.44$ (resp. $c_{\theta_{0}}=0.81$ and $\gamma=0.35$ ) for the experiments (resp. continuum simulations), where $c_{\theta_{0}}$ was obtained by the least squares method keeping the same $\gamma$ than obtained for the flow-rate adjustment in Fig. 11. This observation confirms experimentally that the friction (internal and on the wall) controls the inclination of the streamlines and the presence of a stagnant zone for a horizontal bottom.

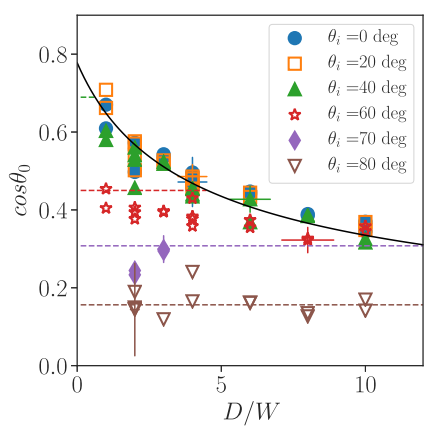

(a)

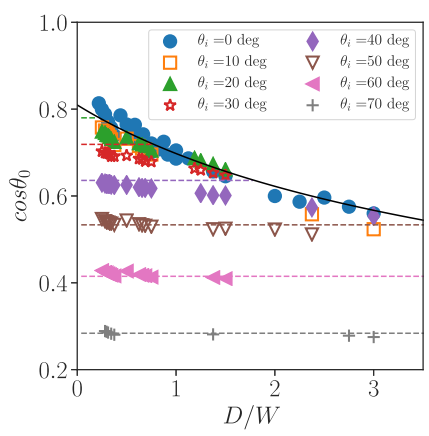

(b)
FIG. 15. Inclination of the central streamline at the outlet, $\cos \theta_{0}$, for all the bottom inclinations vs the aspect ratio of the aperture $D / W$ for (a) the experiments with $d_{p}=538 \mu \mathrm{m}$ and (b) the continuum simulations with $D=0.75 \mathrm{~L}$. The full lines represent Eq. (18) with (a) $c_{\theta_{0}}=0.78$ and $\gamma=0.44$ and (b) $c_{\theta_{0}}=0.81$ and $\gamma=0.35$. The dashed lines represent Eq. (19) with (a) $\zeta=0.9$ and (b) $\zeta=0.83$.

If we suppose that the flow is unperturbed by the bottom while the streamline angle at the center of the outlet generated by the bottom inclination $\theta_{0}$ is smaller than those generated by a horizontal bottom $\theta_{0}^{f}$ we can generalize the model obtained previously for monolayer flow:

$$
\text { if } \begin{gathered}
\theta_{i}<\theta_{c}: \quad \cos \theta_{0}=\cos \theta_{0}^{f}=\frac{c_{\theta_{0}}}{\sqrt{1+\gamma D / W}}, \\
\text { else: } \quad \cos \theta_{0}=\zeta \cos \theta_{i},
\end{gathered}
$$

where we denoted $\theta_{c}$ the critical angle at which the transition occurs:

$$
\theta_{c}=\arccos \left(\frac{c_{\theta_{0}}}{\zeta \sqrt{1+\gamma D / W}}\right) .
$$

In Fig. 15 we compare Eqs. (18) and (19) (black line and dashed lines) with the experimental results. The agreement is fairly good even if this simple model does not finely describe the transition between the two regimes (see, for example, the data for $\theta_{i}=60 \mathrm{deg}$ for the experiments or $\theta_{i}=30 \mathrm{deg}$ for the continuum simulations). This model shows that the transition between a flow controlled by the bottom inclination and a free flow from a lateral aperture strongly depends on $D$ and $W$ on such a confined silo. Again, when the central streamline inclination is controlled by the bottom inclination, the flow rate tends to decrease, the flow being less horizontal.

We now turn to the magnitude of the velocity. In Fig. 16 we study how the magnitude of the velocity of the central streamline at the outlet depends on various parameters. Figure 16(a) corresponds to all the experimental data for $d_{p}=$ $538 \mu \mathrm{m}$ varying the bottom inclination. The data are relatively scattered, but as observe numerically by Zhou et al. [6], the velocity magnitude seems not to depend on the silo thickness and is well adjusted by a square root variation with respect to the opening size given by Eq. (3) with $c_{E}=0.81$. This scaling is not the same as that observed in the 2D case [Eq. (13)]. However, due to the silo confinement, the central streamline is already quite inclined, even for a horizontal bottom [see Fig. 12(a)]. If we plot the data versus $g D \sin \theta_{0}$, the collapse of the data is comparable and the agreement with a square root law [Eq. (13)] is also reasonable with $c_{E}=0.84$ 


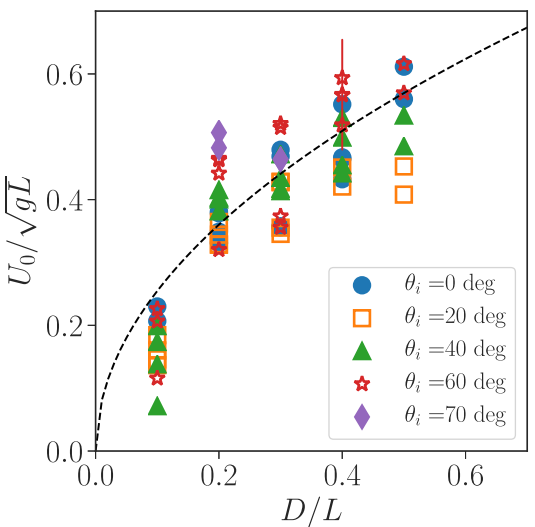

(a)

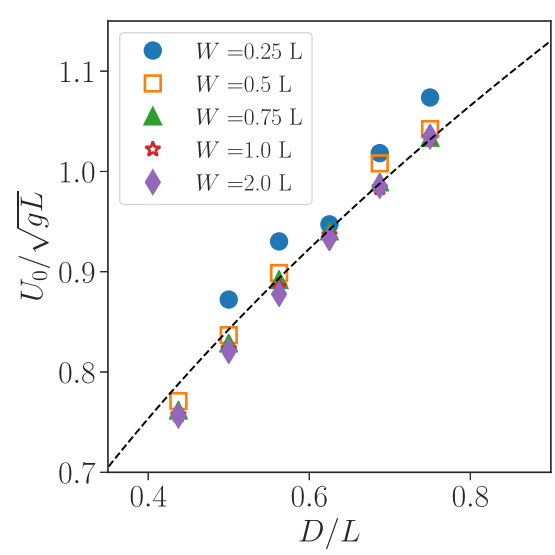

(d)

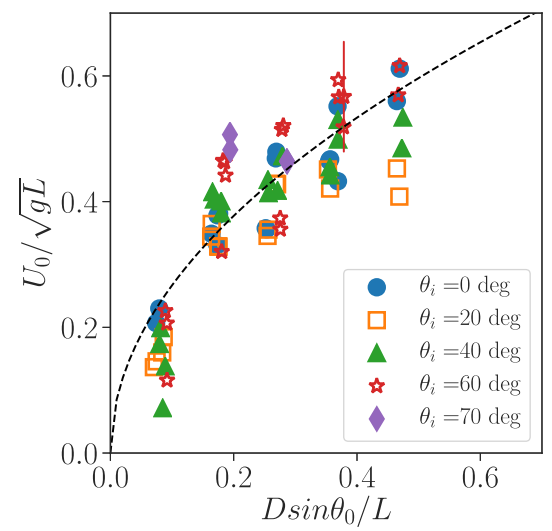

(b)

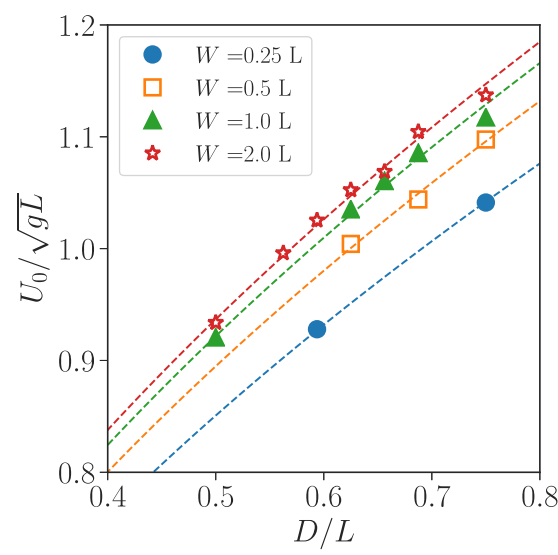

(e)

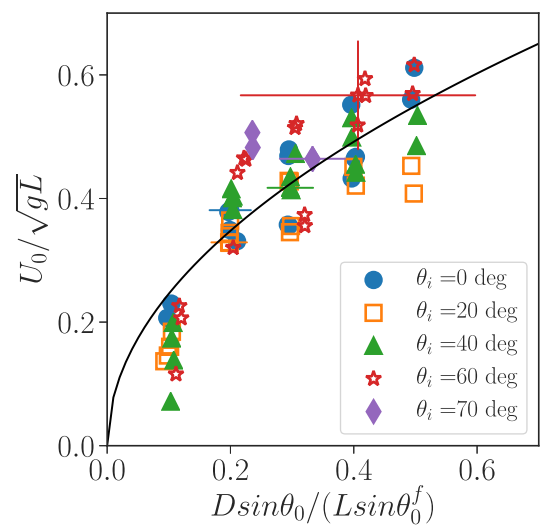

(c)

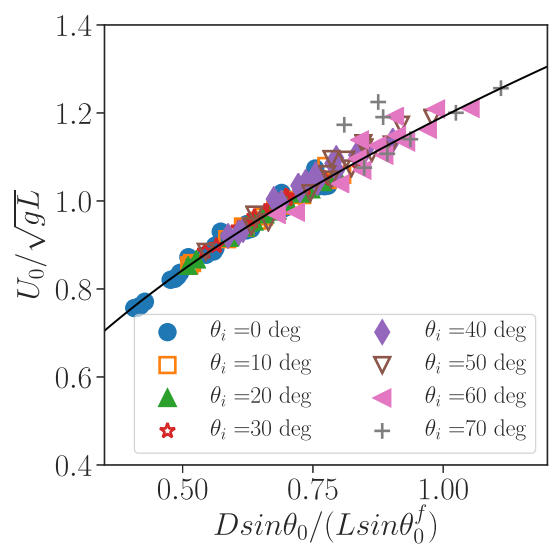

(f)

FIG. 16. Magnitude of the velocity profiles at the center of the outlet, $U_{0}$, normalized by $\sqrt{g L}$ for (a)-(c) the experiments with $d_{p}=538 \mu \mathrm{m}$ and (d)-(f) the continuum simulations vs the outlet $\operatorname{size} D$ normalized by (a), (d), (e) the silo width $L$, (b) $L / \sin \theta_{0}$, (c), (f) $L \sin \theta_{0}^{f} / \sin \theta_{0}$ for (a), (b), (c), (f) various bottom inclination and (d), (e) various silo thicknesses with (d) $\theta_{i}=0$ and (e) $\theta_{i}=40$ deg. The dashed lines represent square root fit with adjustable coefficients obtained by the least squares method. The full lines represent Eq. (21) with (c) $c_{E}=0.78$ and (f) $c_{E}=1.19$.

[see Fig. 16(b)]. This experiment does not allow a conclusion on this point, so we turn to the continuum simulations. For a horizontal bottom we can see in see Fig. 16(d) that, as shown by Zhou et al. [6], the velocity magnitude does not depend on the silo thickness and is well adjusted by a square root variation with respect to the opening size given by Eq. (3) with $c_{E}=1.19$. If we now consider a case where the inclination of the central streamline is controlled by the bottom inclination, as shown in Fig. 16(e) for $\theta_{i}=40^{\circ}$, we can see that the data do not superimpose when varying the silo thickness, the velocity increasing when increasing $W$. If we consider the variation of the magnitude of the velocity with the bottom inclination for a given thickness $W$ (data not shown), we observe that the data are superimposed for low $\theta_{i}$, and then the velocity increases when increasing $\theta_{i}$. However, each curve is well adjusted by a square root variation as given in Eq. (3), the coefficient $c_{E}$ now depending on $W$ and $\theta_{i}$. This suggests that the flow behavior is not the same when the stagnant zone develops freely or when the flow is confined by the inclined bottom. For a free stagnant zone, (i) the parietal friction controls the angle of inclination of the central streamline $\theta_{0}^{f}$ at the outlet, which depends on
$W$ as given by Eq. (18), but (ii) the magnitude of the velocity does not depend on the confinement and is only controlled by the outlet size, giving $U_{0}^{f}=c_{E} \sqrt{g D}$. This may be due to the fact that the stagnant zone is rotating and when reaching the dense granular zone the flow is nearly vertical. When the flow orientation is controlled by the bottom inclination, (i) the flow is not free to rotate, (ii) the inclination at the central streamline is given by Eq. (19), and (iii) the magnitude of the velocity follows $U_{0}=c_{E}^{i} \sqrt{g D \sin \theta_{0}}$. However, Fig. 16(e) suggests that $c_{E}^{i}$ is not constant and depends on $W$. We match both behaviors, writing

$$
U_{0}=c_{E} \sqrt{g D \sin \theta_{0} / \sin \theta_{0}^{f}} .
$$

In Fig. 16(f) the velocity normalized by $\sqrt{g L}$ is plotted versus the normalized outlet size $D \sin \theta_{0} /\left(L \sin \theta_{0}^{f}\right)$. The data are well superimposed and adjusted by Eq. (21) with $c_{E}=1.19$. This observation suggests that when the flow orientation is controlled by the bottom inclination, the parietal friction still played a role controlling the magnitude of the velocity at the outlet. This effect will tend to counterbalance the decrease of 


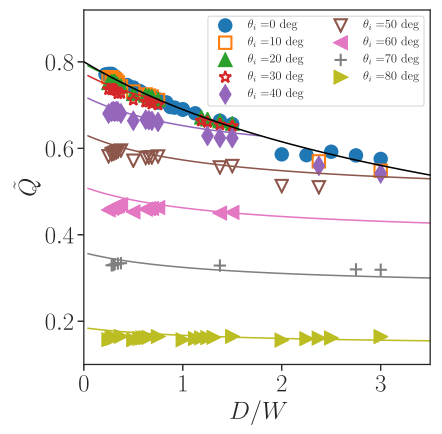

(a)

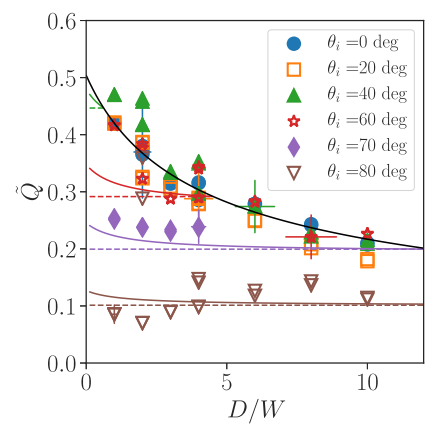

(b)
FIG. 17. Mass flow rate normalized by $\rho_{p} \phi_{b} D W \sqrt{g D}$ for all the bottom inclinations vs the normalized aperture size $D / W$ for (a) the continuum simulations and (b) the experiments with $d_{p}=538 \mu \mathrm{m}$. The full black lines represent Eq. (22), the full color lines represent Eq. (23), and the dashed lines represent Eq. (15) with (a) $c_{D}=0.8$, $c_{\theta_{0}}=0.81, \gamma=0.35$, and $\zeta=0.83$ and (b) $c_{D}=0.5, c_{\theta_{0}}=0.78$, $\gamma=0.44$, and $\zeta=0.9$.

the flow rate a little bit due to the strong inclination of the streamlines. In Fig. 16(c) we plot the data corresponding to the experiments with $d_{p}=538 \mu \mathrm{m}$ in the same representation. The collapse of the data and the agreement with Eq. (21) with $c_{E}=0.78$ is equivalent to that previously found in Figs. 16(a) and $16(\mathrm{~b})$.

We can then generalize the analytical model, writing

$$
\begin{gathered}
\text { if } \theta_{i}<\theta_{c}: \quad \tilde{Q}=\frac{c_{D}}{\sqrt{1+\gamma D / W}}, \\
\text { else: } \tilde{Q}=c_{\theta} \cos \theta_{i}\left[\frac{\left(1-\zeta^{2} \cos ^{2} \theta_{i}\right)(1+\gamma D / W)}{1-c_{\theta_{0}}^{2}+\gamma D / W}\right]^{\frac{1}{4}}
\end{gathered}
$$

with $\tilde{Q}=Q /\left(\rho_{p} \phi_{b} D W \sqrt{g D}\right), c_{D}=c_{0} c_{E} c_{\theta_{0}}$, and $c_{\theta}=c_{0} c_{E} \zeta$. When the silo is very thin (i.e., $W \ll D$ ), Eq. (23) reduces to Eq. (15) corresponding to the monolayer case and to Eq. (16) for inclination angles larger than $40^{\circ}$ with an error less than $15 \%$. To test this model, we can first compare the coefficient $c_{D}=0.5$ (resp. 0.8) adjusted on the flow rate for the horizontal bottom and the value of $c_{0} c_{E} c_{\theta_{0}}=0.48$ (resp. 0.85) obtained from the velocities measurement, which shows good agreement in the experiments (respectively the continuum simulations). Then in Fig. 17 we compare the model given by Eqs. (22) and (23) with the mass flow rate normalized by $\rho_{p} \phi_{b} D W \sqrt{g D}$ plotted versus the normalized aperture $D / W$, both for the simulations and the experiments. For the continuum simulations [Fig. 17(a)] the agreement is very good. For large bottom inclination $\left(\theta_{i}>50 \mathrm{deg}\right)$ the flow rate is completely controlled by the bottom, whereas for small bottom inclination $\left(\theta_{i}<20 \mathrm{deg}\right)$ it is controlled by the friction (internal to the granular medium and with the walls). In between, even when the inclination of the streamline is controlled by the bottom, the model predicts that the flow rate stays close to the unperturbed flow. For the experiments [Fig. 17(b)] the agreement is also fairly good for large bottom inclination $\left(\theta_{i}>70 \mathrm{deg}\right)$ or small bottom inclination $\left(\theta_{i}<40 \mathrm{deg}\right)$, but the model fails to describe the data close to the transition between the two regimes. In particular, it is interesting to note that for $\theta_{i}=60 \mathrm{deg}$ the flow rate is superimposed to the
TABLE III. Experimentally measured fitting parameters with associated equation number: $\gamma(5), c_{D}(4), c_{\theta_{0}}(5), c_{E}(21), \zeta(12)$, and $c_{\theta}$ (15).

\begin{tabular}{lcccccccc}
\hline \hline$d_{p}(\mu \mathrm{m})$ & $c_{D}$ & $\gamma$ & $c_{\theta_{0}}$ & $c_{E}$ & $\zeta$ & $c_{0}$ & $c_{0} c_{E} c_{\theta_{0}}$ & $c_{\theta}$ \\
\hline $4170(2 \mathrm{D})$ & 0.5 & - & 0.63 & 1.29 & 0.92 & 0.89 & 0.71 & 1.04 \\
1347 & 0.41 & 0.28 & 0.81 & 0.94 & 0.92 & 0.85 & 0.65 & 0.74 \\
1129 & 0.45 & 0.44 & 0.86 & 0.92 & 0.92 & 0.81 & 0.64 & 0.69 \\
762 & 0.42 & 0.28 & 0.69 & 0.95 & 0.92 & 0.81 & 0.53 & 0.71 \\
538 & 0.5 & 0.44 & 0.78 & 0.84 & 0.9 & 0.79 & 0.52 & 0.60 \\
375 & 0.52 & 0.53 & 0.81 & 0.84 & 0.85 & 0.83 & 0.56 & 0.59 \\
\hline
\end{tabular}

unperturbed flow rate, whereas the inclination of the central streamline is already perturbed [see the red stars in Figs. 17(b) and 15(a)]. We also observe that the simplified model corresponding to Eq. (15) adjusts the data quite well (see the dashed lines).

Even if this simple model does not intricately capture this particular behavior, it succeeds to predict the flow rate in the two asymptotic regimes where the flow is controlled either by the silo confinement through the wall friction or by the inclined bottom. Moreover, the continuum model appears to reproduce the experimental behavior quite well, except at the transition between the two regimes. This may be due to the choice of the no-slip condition at the bottom, which may be less realistic when the flow is perturbed by the inclined bottom wall. In the next section we will compare the analytical model with the other particles studied.

\section{Comparisons and discussion}

In Table III we have summed up the fitting parameters deduced from the balance measurement and image processing for all particles large enough to perform accurate particle image velocimetry $\left(d_{p} \geqslant 375 \mu \mathrm{m}\right)$. For particles larger than $1000 \mu \mathrm{m}$ we obtain that $c_{0} c_{E} c_{\theta_{0}}$ slightly overevaluates the value of $c_{D}$. We interpret such behavior as being related to the small dilatation of the particles at the outlet, associated with a low number of particles through the outlet. We observe that except for the monolayer case, the parameters obtained from the image processing seem to be rather constant, with $c_{\theta_{0}} \approx 0.8, c_{E} \approx 0.9, \zeta \approx 0.9, c_{0} \approx 0.8$.

To evaluate the evolution of the flow rate with the bottom inclination using the simplified Eq. (15), four parameters are necessary: $c_{D}, \gamma, c_{\theta}$, and $\zeta$. We can deduce the two first using flow-rate measurements with a horizontal bottom. From Table III we evaluate $c_{\theta} \approx 0.7$ and $\zeta \approx 0.9$. In Fig. 18 we have plotted the flow rate for the smallest particles $d_{p}=190 \mu \mathrm{m}$, for which we have not performed image processing, and compared it with the analytical model given by Eqs. (22) and (15). We recover a good agreement for the asymptotic regimes (small and large angle) and a discrepancy at the transition for $\theta_{i}=60 \mathrm{deg}$, where the model predicts a constant flow rate for low $D / W$, whereas the flow rate continues to follow the same variation than for a horizontal bottom.

This simple phenomenological model, which depends on four fitting parameters, allows us to recover the main feature of the dependence of the flow rate with the bottom inclination in a rectangular silo with a lateral orifice. 


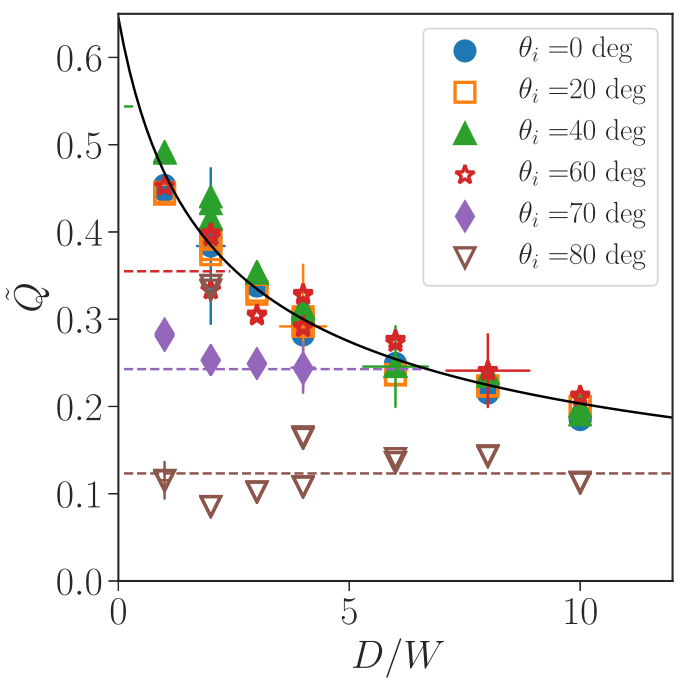

FIG. 18. Experiments with $d_{p}=190 \mu \mathrm{m}$ : Mass flow rate normalized by $\rho_{p} \phi_{b} D W \sqrt{g D}$ for all the bottom inclinations vs the normalized aperture size $D / W$. The full line represents Eq. (22) and the dashed lines Eq. (15) with $c_{D}=0.65, \gamma=0.91, \xi_{\theta}=1.1$.

\section{CONCLUSION AND PERSPECTIVES}

Using a nonconventional geometry (a rectangular silo with an inclined bottom wall which ends up at a lateral orifice) we have shown, thanks to laboratory experiments, contact dynamic simulations and a continuum model with a viscoplastic frictional rheology $\mu(I)$ that the flow rate is controlled by two phenomena. First the orientation of the granular flow is controlled by a competition between the friction terms and the silo geometry. For small angles of inclination of the bottom, the flow is not perturbed by the geometry and the central streamline orientation is controlled by the outlet aspect ratio
$D / W$ resulting from the competition between wall friction and internal friction. For large angles the flow orientation is imposed by the bottom inclination. Then we have shown that the outlet corresponds to an area where the grains are accelerated, the potential energy being transferred to kinetic energy. The magnitude of the velocity still seems to depend on the internal and wall friction for high bottom inclination. However, this balance and thus the magnitude of the velocity at the center of the outlet is found to be quite sensitive to the boundary conditions. Finally, we present a simple analytical model that predicts the discharge flow rate of particles from a rectangular silo with an inclined bottom according to its aperture aspect ratio with a good agreement in the asymptotic regime and a small discrepancy in the transition zone.

The discrete simulation results should be enhanced, of course, by fully 3D computations, but first with more study of friction between grains and with a better description of the lateral friction in the monolayer case. The continuum modeling is shown to be a good framework to describe the discharge flow of a granular medium from a silo, and in future work it will allow the performance of a full parametric study of the influence of the rheological parameters on the adjustable parameters of the analytical model in various geometries. In particular, these results may have practical interest when considering the effects of the hopper angle of an industrial silo.

\section{ACKNOWLEDGMENTS}

This work is funded by the Institut de Radioprotection et de Sûreté Nucléaire (IRSN) and Électricité de France (EDF) under their collaborative research programs. It has been carried out within there framework of the Labex MEC (ANR-10-LABX-0092) under the A*MIDEX project (ANR-11-IDEX-0001-02) funded by the French government program Investissements d'Avenir.
[1] G. H. L. Hagen, Bericht über die zur Bekanntmachung geeigneten Verhandlungen der Königlich Preussischen Akademie der Wissenschaften zu Berlin, 35 (1852).

[2] B. Tighe and M. Sperl, Granular Matter 9, 141 (2007).

[3] W. A. Beverloo, H. A. Leniger, and J. V. de Velde, Chem. Eng. Sci. 15, 260 (1961).

[4] A. Janda, I. Zuriguel, and D. Maza, Phys. Rev. Lett. 108, 248001 (2012).

[5] M. Benyamine, M. Djermane, B. Dalloz-Dubrujeaud, and P. Aussillous, Phys. Rev. E 90, 032201 (2014).

[6] Y. Zhou, P.-Y. Lagrée, S. Popinet, P. Ruyer, and P. Aussillous, J. Fluid Mech. 829, 459 (2017).

[7] S. M. Rubio-Largo, A. Janda, D. Maza, I. Zuriguel, and R. C. Hidalgo, Phys. Rev. Lett. 114, 238002 (2015).

[8] P. Jop, Y. Forterre, and O. Pouliquen, Nature (London) 441, 727 (2006).

[9] L. Staron, P.-Y. Lagrée, and S. Popinet, Phys. Fluids 24, 103301 (2012).

[10] S. Dunatunga and K. Kamrin, J. Fluid Mech. 779, 483 (2015).
[11] G. Davier and F. Bertails-Descoubes, J. Non-Newt. Fluid Mech. 234, 15 (2016).

[12] H. G. Sheldon and D. J. Durian, Granular Matter 12, 579 (2010).

[13] P. Meunier and T. Leweke, Exp. Fluids 35, 408 (2003).

[14] F. Dubois, M. Jean, M. Renouf, R. Mozul, A. Martin, and M. Bagneris (private communication).

[15] F. Radjai and F. Dubois, Discrete-Element Modeling of Granular Materials (Wiley-Iste, Berlin, 2011), p. 425.

[16] Y. Zhou, P. Ruyer, and P. Aussillous, Phys. Rev. E 92, 062204 (2015).

[17] G. D. R. MiDi, Eur. Phys. J. E 14, 341 (2004).

[18] P. Jop, Y. Forterre, and O. Pouliquen, J. Fluid Mech. 541, 167 (2005).

[19] Y. Forterre and O. Pouliquen, Annu. Rev. Fluid Mech. 40, 1 (2008).

[20] L. Staron, P.-Y. Lagrée, and S. Popinet, Eur. Phys. J. E 37, 5 (2014).

[21] R. Artoni, A. Santomaso, and P. Canu, Europhys. Lett. 80, 34004 (2007). 
[22] T. Barker, D. G. Schaeffer, P. Bohorquez, and J. M. N. T. Gray, J. Fluid Mech. 779, 794 (2015).

[23] P.-P. Cortet, D. Bonamy, F. Daviaud, O. Dauchot, B. Dubrulle, and M. Renouf, Europhys. Lett. 88, 14001 (2009).
[24] S. Popinet, Basilisk C reference manual (2013-2016), http: //basilisk.fr/Tutorial.

[25] See Supplemental Material at http://link.aps.org/supplemental/ 10.1103/PhysRevE.102.052902 for experimental and numerical data. 